University of Nebraska - Lincoln

DigitalCommons@University of Nebraska - Lincoln

Papers in the Earth and Atmospheric Sciences

Earth and Atmospheric Sciences, Department

\title{
Diatom biostratigraphy of the Cenozoic glaciomarine Pagodroma Group, northern Prince Charles Mountains, East Antarctica
}

\author{
Jason M. Whitehead \\ University of Tasmania, jason.whitehead@utas.edu.au \\ David M. Harwood \\ University of Nebraska-Lincoln, dharwood1@unl.edu \\ Barrie McKelvey \\ University of New England, Armidale, Australia, bmckelve@une.edu.au \\ Andrew McMinn \\ University of Wales, Andrew.McMinn@utas.edu.au
}

Follow this and additional works at: https://digitalcommons.unl.edu/geosciencefacpub

Part of the Earth Sciences Commons

Whitehead, Jason M.; Harwood, David M.; McKelvey, Barrie; and McMinn, Andrew, "Diatom biostratigraphy of the Cenozoic glaciomarine Pagodroma Group, northern Prince Charles Mountains, East Antarctica" (2004). Papers in the Earth and Atmospheric Sciences. 283.

https://digitalcommons.unl.edu/geosciencefacpub/283

This Article is brought to you for free and open access by the Earth and Atmospheric Sciences, Department of at DigitalCommons@University of Nebraska - Lincoln. It has been accepted for inclusion in Papers in the Earth and Atmospheric Sciences by an authorized administrator of DigitalCommons@University of Nebraska - Lincoln. 
Published in Australian Journal of Earth Sciences 51: 4 (2004), pp. 521 - 547; doi: 10.1111/j.1400-0952.2004.01072.x

Copyright ( 92004 Geological Society of Australia; published by Taylor \& Francis Ltd. Used by permission.

Submitted September 17, 2003; accepted June 8, 2004; published online December 23, 2004.

\title{
Diatom biostratigraphy of the Cenozoic glaciomarine Pagodroma Group, northern Prince Charles Mountains, East Antarctica
}

\author{
J. M. Whitehead, ${ }^{1,2}$ D. M. Harwood, ${ }^{2}$ B. C. McKelvey, ${ }^{3}$ M. J. Hambrey, ${ }^{4}$ A. McMinn ${ }^{1}$ \\ 1. Institute of Antarctic and Southern Ocean Studies, University of Tasmania, Tas. 7005, Australia \\ 2. Department of Geosciences, University of Nebraska-Lincoln, Lincoln, NE, USA \\ 3. Division of Earth Sciences, University of New England, Armidale, NSW 2351, Australia \\ 4. Centre for Glaciology, Institute of Geography and Earth Sciences, University of Wales, Aberystwyth, Ceredigion SY23 3DB, UK
}

Corresponding author - J. M. Whitehead, email jason.whitehead@utas.edu.au

\begin{abstract}
In the northern Prince Charles Mountains glaciomarine sediments of the Pagodroma Group outcrop on Fisher Massif (Mt Johnston and Fisher Bench Formations) and at the Amery Oasis (Battye Glacier and Bardin Bluffs Formations), at locations 300 and $250 \mathrm{~km}$ south of the Amery Ice Shelf edge, respectively. Most of the Pagodroma Group consists of ice-proximal glaciomarine diamict, and a much subordinate $(<2 \%)$ amount of more ice-distal mudstone. Microfossil biostratigraphy based upon in situ and glacially reworked diatoms constrains the ages of the four formations, and identifies at least six intervals of marine fjordal deposition. Sparse diatoms in Mt Johnston Formation diamicts indicate an Early Oligocene age. However, it is unclear whether these diatoms are in situ or else are glacially reworked and represent either an immediately prior or more remote past interglacial. The Battye Glacier and Fisher Bench Formations contain in situ diatoms and are broadly time equivalent, the former dating between 10.7 and 9.0 Ma and the latter between 12.1 or $10.7 \mathrm{Ma}$ and $8.5 \mathrm{Ma}$. In situ diatoms indicate the Bardin Bluffs Formation to have been deposited between 2.6 and either 1.8 or $0.99 \mathrm{Ma}$. Glacially reworked diatoms in the Bardin Bluffs and Fisher Bench Formations identify four depositional intervals. The reworked taxa are sourced from EoceneOligocene (>30.1 Ma), Middle Miocene (14.5-12.5 Ma and 12.1-11.5 Ma), Early Pliocene (4.9-3.6 Ma) and, tentatively, Late Pliocene (3.4-2.6 Ma) strata. These microfossil data further develop the interpretation that the Pagodroma Group formed during episodes of reduced glacial extent when, in the absence of an ice shelf, marine waters penetrated far southwards into the Lambert Graben of East Antarctica.
\end{abstract}

Keywords: Antarctic Ice Sheet, Cenozoic, diatoms, Pagodroma Group, Prince Charles Mountains

\section{Introduction}

This paper documents siliceous microfossil occurrences in the Cenozoic Pagodroma Group of the northern Prince Charles Mountains (McKelvey et al. 2001) in East Antarctica, and assesses the biostratigraphic ages of the strata. The research comprises both new data and some reassessment of previous studies. In addition, diatom assemblage composition, preservation and abundance are used to critically assess the mode of microfossil emplacement. Conceivably the Pagodroma Group microfossils represent (i) in situ primary deposition, (ii) aeolian contamination, (iii) glacial or other reworking processes. Understanding the precise mode of microfossil emplacement is crucial to the accurate biostratigraphic age assessment of the Pagodroma Group.

\section{Location and geological setting}

The largest single catchment area of the East Antarctic Ice Sheet $(\sim 7 \%)$ drains through the Lambert Graben (Hambrey \& Dowdeswell 1994), which is now covered by the Amery Ice Shelf (Figure 1). This ice shelf grounds along the sides of the graben, and extends nearly $500 \mathrm{~km}$ southwestwards from the open sea in Prydz Bay (Phillips et al. 1996; Krebs 1997, 1998). The largely ice-free Amery Oasis and the nunatak Fisher Massif, both in the northern Prince Charles Mountains on the western side of the Lambert Graben, are situated $\sim 250 \mathrm{~km}$ and $\sim 300 \mathrm{~km}$ inland from the seaward edge of the Amery Ice Shelf, respectively (Figure 1). The northern Prince Charles Mountains consist primarily of Proterozoic high-grade metamorphic rocks (Tingey 1991; Mikhalsky et al. 2001). However, Permo-Triassic fluvial strata of the Amery Group also occur in the Amery Oasis (Fielding \& Webb 1995; McLoughlin \& Drinnan 1997).

Appendices 1 and 2 are indicated by an asterisk $\left({ }^{*}\right)$ in the text and are printed at the end of the paper. They are also published as Supplementary Papers, and copies may be obtained from the Geological Society of Australia's website at http://www.gsa.org.au or from the National Library of Australia's Pandora archive at http://nla.gov.au/nla.arc-25194. 
Cenozoic glaciomarine strata of the Pagodroma Group extensively cover the Amery Oasis and Fisher Massif, and outcrop in cliff sections of up to $300 \mathrm{~m}$, with a composite record totaling more than $800 \mathrm{~m}$ in thickness in the northern Prince Charles Mountains (McKelvey et al. 2001). The Pagodroma Group comprises at least four geographically separated formations: the Mt Johnston and Fisher Bench Formations occur on Fisher Massif, and the Battye Glacier and Bardin Bluffs Formations are distributed about the Radok Lake - Beaver Lake region of the Amery Oasis (Figures 2,3) (Hambrey \& McKelvey 2000a; McKelvey et al. 2001; Whitehead \& McKelvey 2001). These Cenozoic strata consist of massive and stratified diamict, boulder gravel, and minor laminated sandstone and mudstone, and are interpreted as fjordal deposits that accumulated seawards of tidewater glacier fronts in environments akin to the modern fjords of East Greenland (Hambrey \& McKelvey 2000a, b). Each formation was deposited in the Lambert Graben during a phase of glacial recession and marine incursion. Subsequent glacial advances largely eroded the formations (and the underlying basement), and redeposited the sediments on the continental shelf and slope of Prydz Bay (Barron et al. 1991; Barker et al. 1998; O'Brien et al. 2001). The Pagodroma Group outcrops are erosional remnants that now occupy the floors and abut the walls of uplifted former Lambert Graben fjords (Figure 4). Because of this uplift the older Pagodroma Group formations occupy the higher elevations (McKelvey et al. 2001; Hambrey \& McKelvey 2000a Table 1). Given the known rates of modern east Greenland fjordal sedimentation, it is apparent that the individual formations represent time intervals in the order of only hundreds or thousands of years (Dowdeswell et al. 1994). That this stratigraphic record spans an interval of perhaps 35 million years emphasises its extremely fragmentary nature.

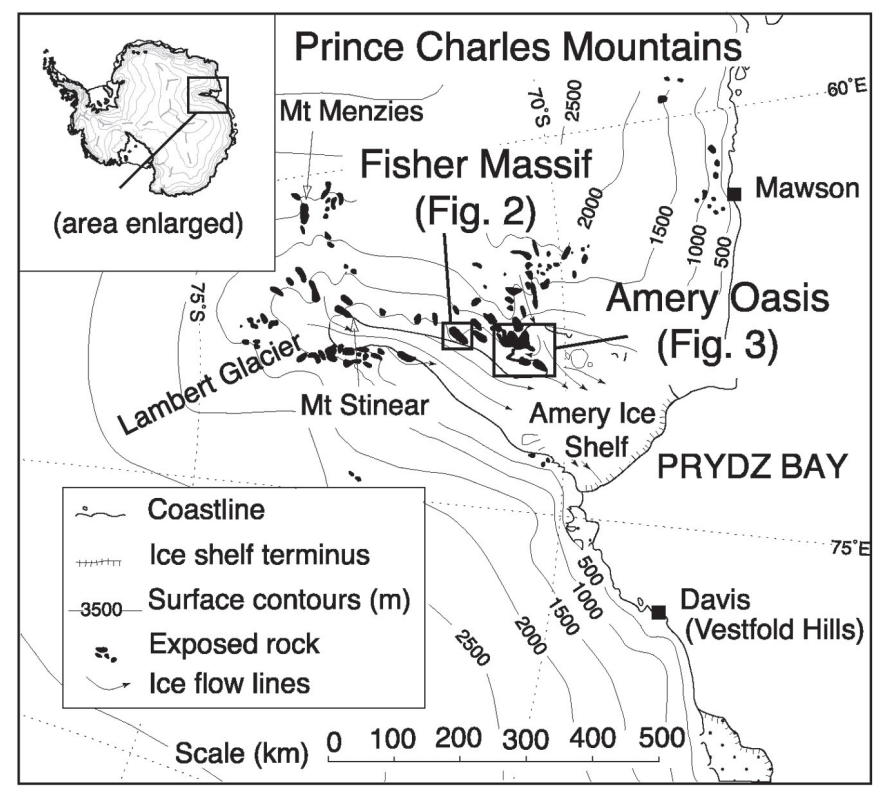

Figure 1. The Prince Charles Mountains showing positions of Fisher Massif and Amery Oasis within the Lambert Glacier - Amery Ice Shelf drainage system. Inset: Location of study area in Antarctica.

\section{Previous work}

Cenozoic marine diatoms are known from the Bardin Bluffs Formation (Bardin \& Belevich 1985; McKelvey \& Stephenson 1990; Whitehead \& McKelvey 2001), the Fisher Bench Formation (Laiba \& Pushina 1997; McKelvey et al. 2001), and the Battye Glacier Formation (Whitehead et al. 2003) of the Pagodroma Group. Other microfossils recovered include foraminifers, ebridians, silicoflagellates, sponge spicules and a radiolarian (McKelvey et al. 2001; Whitehead et al. 2003). Permo-Triassic spores and pollen are also present (Bardin $\&$ Belevich 1985). These microfossils have been recovered from the mudstone facies and, to a lesser degree, from the varied diamicts. The most diatom-rich mudstone noted so far, in which biogenic opal comprises up to $12 \%$ of the sediment (by dry weight), occurs in the Battye Glacier Formation (Whitehead et al. 2003).

\section{Methods}

Twenty-seven samples of the Pagodroma Group from Amery Oasis and Fisher Massif were collected by McKelvey, Hambrey and Whitehead during Australian National Antarctic Research Expeditions (ANARE) in 1987/88, 1989/90, 1994 /95, and 1997/98. Additional data reported by Bardin and Belevich (1985), Laiba and Pushina (1997) and Whitehead et al. (2003) are also included in the present study. The geographical and stratigraphic positions of the samples are indicated in Figures 2, 3. In Figure 3d only the approximate position of Bardin Bluffs "Top" and "Bottom" samples can be indicated. Sample lithologies are recorded as diamict (Dm) or mudstone (Mud.) (Table 1). More detailed lithological information of sampled intervals exists in Laiba and Pushina (1997), Hambrey and McKelvey (2000a), Whitehead and McKelvey (2001), and Whitehead et al. (2003). Samples considered in the latter two publications were collected from $>10 \mathrm{~cm}$-deep excavations into outcrops, to avoid possible outcrop surface contamination by airborne diatoms.

Initially strewn microfossil slides were prepared from raw (unprocessed) samples (so indicated in Table 1), mounted with Norland optical adhesive \#61 (refractive index = 1.56), and studied with an Olympus BH-2 light microscope at low magnification $(\times 600)$ and at high magnification $(\times 1000)$ for taxonomic identification (Figures 5, 6; Appendix 1*). Those raw samples with only sparse or else no apparent diatoms visible were processed further in the University of Nebraska's geological clean laboratory that is dedicated to microfossil extraction from glacial deposits. Here microfossils in these samples were concentrated using elutriation, sieving, gravity-settling and heavy-liquid density separation (using sodium polytungstate), as described in Harwood and Rose (1998). Such samples are recorded as "concentrated" in Table 1, and were studied with a light microscope in the same manner as the "raw" slides.

Quantitative abundance data were collected by systematically scanning microfossil slides. The use of raw and concentrated slides, and the typically fragmented nature of the fossils, precluded the collection of absolute abundance data. Therefore, qualitative indices ranging from 0 to 6 were used 
to describe the slide fossil abundances (Table 1): 0, no specimens or fragments observed; 1 , at least one specimen or fragment per slide; 2 , a few specimens per slide; 3 , numerous specimens per slide $<10 ; 4$, many specimens per slide $>10 ; 5$, a specimen or fragment every few fields of view $(\times 600$ magnification); and 6, numerous specimens or fragments on every field of view (×600 magnification). In selected samples the size and abundance of fragments, and the occurrence of whole diatoms and diatomaceous clasts were recorded (Appendix $2^{*}$ ). To further quantify the microfossils, several samples with the highest diatom abundance as seen with the light microscope were analysed for biogenic silica using a SHIMATZU UV1201 spectrophotometer, following the methods of Mortlock and Froelich (1989), at the Antarctic Cooperative Research Centre, Tasmania. This method allows quantification of the siliceous microfossil content as a percentage of the sediment (dry weight) and is referred to as biogenic silica (\%opal) (Table 1).

The diatom biostratigraphy of the Pagodroma Group (Figures 7,8 ) has been undertaken using established data (Table 2) calibrated to the Berggren et al. (1995) geomagnetic polarity time-scale. Descriptive criteria of the microfossil occurrences, assemblage composition and macrofossil association have been tabulated to help assess the mode of fossil emplacement (Table 3).

\section{Results}

Siliceous microfossils have been identified in 22 of the 27 samples examined. Diatom abundances vary, but are generally considerably higher in mudstone rather than diamict samples (Table 1). The highest diatom occurrences noted are in raw slides prepared from mudstone samples AM-015 and PCM 98-3Aa-26 of the Fisher Bench and Bardin Bluffs Formations, respectively. Diamict samples yielded only low abundances or else are barren (Table 1). The size and preservation of different fossil elements also varies between samples (Appendix 2*). Extant and extinct planktic and benthic marine, and freshwater taxa have been identified. The assemblages largely consist of planktic marine species, but several samples are rich in benthic marine taxa (e.g. sample PCM 98-3Aa-26 of the Bardin Bluffs Formation).

\section{Mt Johnston Formation}

Mt Johnston Formation diamicts (Figures 2, 3) (samples PCM 90-4, PCM 90-5, AM-003, AM-006, and AM-014) contain very sparse extinct planktic marine diatoms and diatom fragments (Table 1), including the species Coscinodiscus sp., Pyxilla sp., Stephanopyxis grunowii Grove \& Sturt, Stephanopyxis splendidus (Greville) Harwood and Thalassiothrix/Thalassionema spp.

\section{Fisher Bench Formation}

Mudstone and diamict facies of the Fisher Bench Formation (Figures 2,3) contain marine diatoms and diatom fragments (Table 1). Abundant diatoms occur in mudstone intercalated within diamict at the base of the section (sample AM-015).
These diatoms are moderately well-preserved; at least half consist of intact valves, and a very few complete frustules are present. Occasionally the valves are filled with terrigenous mud, similar in appearance to the supporting matrix. During previous studies of sample AM-015 no archive material was retained for biogenic silica analysis; however, a visual estimate of $\sim 2 \%$ is suggested through comparison with diatom abundance in the raw slide of sample PCM 98-3Aa26 from the Bardin Bluffs Formation (Table 1). The AM-015 diatom assemblage consists predominately of planktic marine species, benthic diatoms constituting $<1 \%$ of the total relative abundance. Much of the assemblage is dominated by the extinct Actinocyclus ingens Rattray, and to a lesser degree by the extant taxon, Coscinodiscus sp. A, Coscinodiscus radiatus Ehrenberg and the extinct Denticulopsis vulgaris (Okuno) Yanagisawa \& Akiba. Another mudstone, $\sim 66 \mathrm{~m}$ above sample AM-015, contains a few planktic diatom fragments of Thalassiothrix spp. (sample PCM 90-6). At approximately the same horizon as sample PCM 90-6, Laiba and Pushina (1997) identified a diatom-rich mudstone (sample 36721-4), which contains a predominantly benthic marine assemblage: Hyalodiscus obsoletus Sheshukova, Rhabdonema sp. and Rhaphoneis spp. Some rarer planktic taxa such as Denticulopsis simonsenii Yanagisawa \& Akiba were also identified.

Diamict sample PCM 90-11 (Figure 2c) contains a diatom assemblage that differs bimodally in preservation and composition (Appendix $2^{*}$ ). The sample yielded an assemblage of well-preserved (intact frustules) benthic diatoms: Gomphonema angustatum var. sp. A, G. angustatum var. sp. B, Diploneis sp. A and Amphora sp. A. The remainder of the assemblage consists largely of planktic marine diatom fragments, notably Chaetoceros sp. A seta, Thalassiosira spp. and rare occurrences of other taxa (Table 1).

\section{Battye Glacier Formation}

Diatom occurrences are rare in both the diamict and mudstone facies of the Battye Glacier Formation type section at the Dragons Teeth (samples PCM 90-8 and PCM 90-10B) (Figure 3c Section 1). Fragments of Thalassiothrix sp. and a sponge spicule have been recovered (Table 1). However, Whitehead et al. (2003) described exceptionally diatom-rich intervals (up to $12 \%$ biogenic opal, sediment dry weight) in the Battye Glacier Formation some $13 \mathrm{~km}$ to the northeast of the Dragons Teeth, above the western shore of Beaver Lake (Figure 3c Section 2). Here the assemblages in samples PCM 00-116 and PCM 00-118 are rich in benthic diatoms, although planktic taxa such as D. vulgaris are present. The Battye Glacier Formation facies at Beaver Lake represent a different (more basinal) glacigene palaeoenvironment to that at Dragons Teeth (Whitehead et al. 2003).

\section{Bardin Bluffs Formation}

The basal Bardin Bluffs Formation (Figure 3d) consists of two members (Whitehead \& McKelvey 2001). The older Member 1 , consists largely of mudstone and muddy sandstone, both of which contain abundant dropstones. Member 2, which consists essentially of diamict, disconformably overlies Member 1. Diatoms occur in both members (Table 1). 


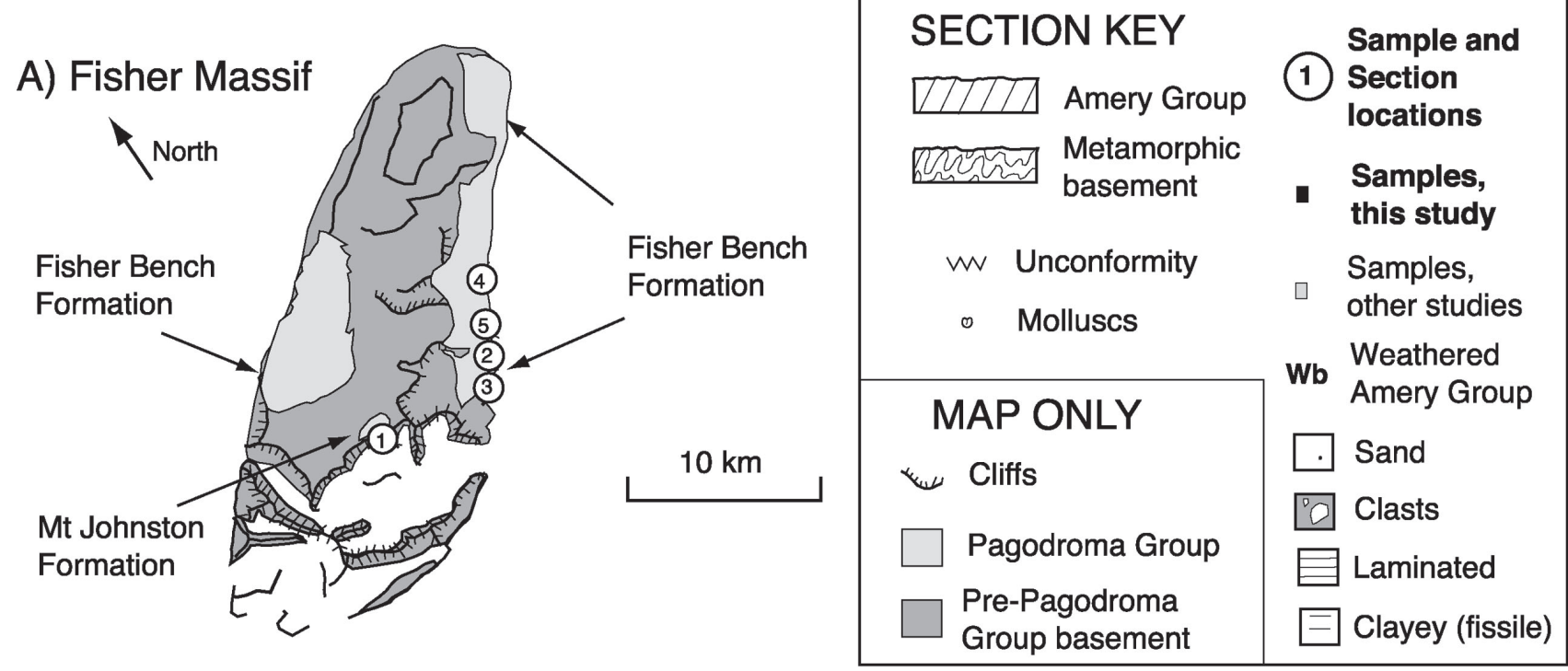

Other Fisher Bench Formation samples

C) Fisher Bench Formation
(4) AM-026
(5)
AM-017
B) Mt Johnston Formation
(1) Section
(71'31'43"S; $\left.67^{\circ} 34^{\prime} 43^{\prime \prime E}\right)$

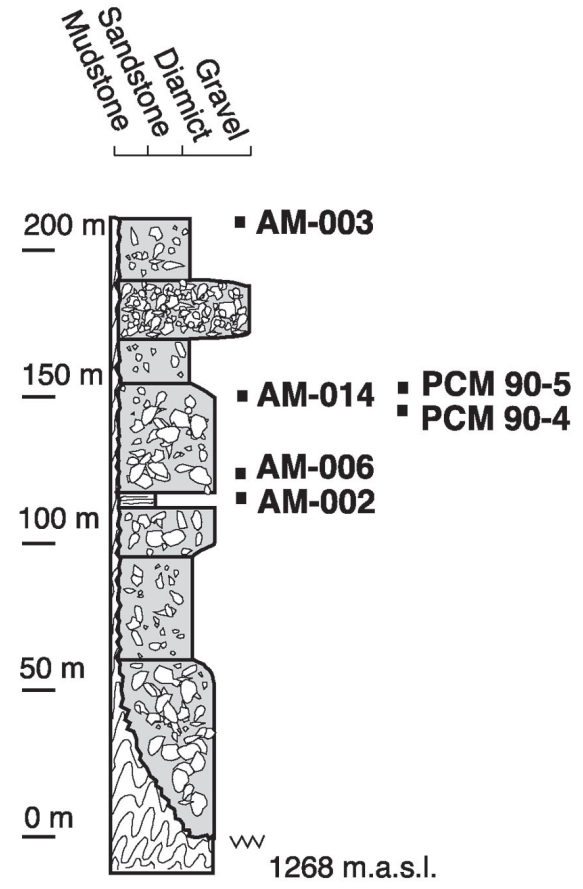

(3) Section (Laiba and Pushina 1997)
(713'ㄴ' $\left.51^{\prime \prime S} ; 67^{\circ} 43^{\prime 2} 23^{\prime \prime} E\right)$

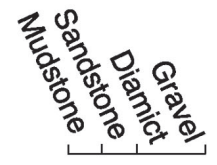

$\underline{300 \mathrm{~m}}$

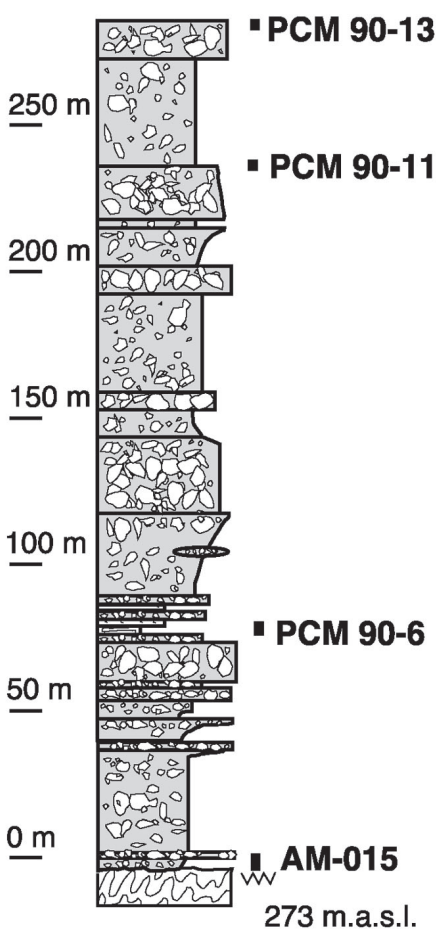

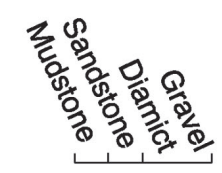

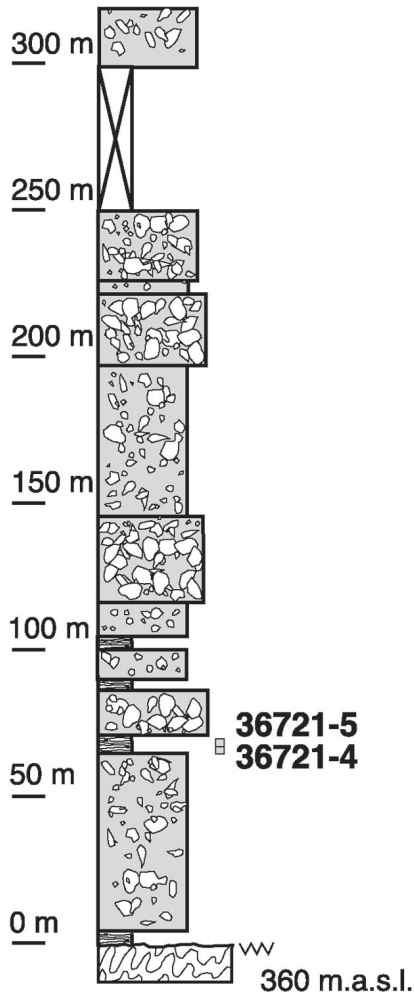

Figure 2. (a) Location of Pagodroma Group sections at Fisher Massif. (b) Mt Johnston Formation and (c) Fisher Bench Formation sections and sample positions. Sections 1 and 2 are from Hambrey and McKelvey (2000a) and section 3 is from Laiba and Pushina (1997). Gravel and diamict are distinguished from each other texturally, and not by clast/grain size as suggested here. The stratigraphic position of samples analysed in the present study are indicated by black boxes and samples from other studies are indicated by grey boxes (see key). 

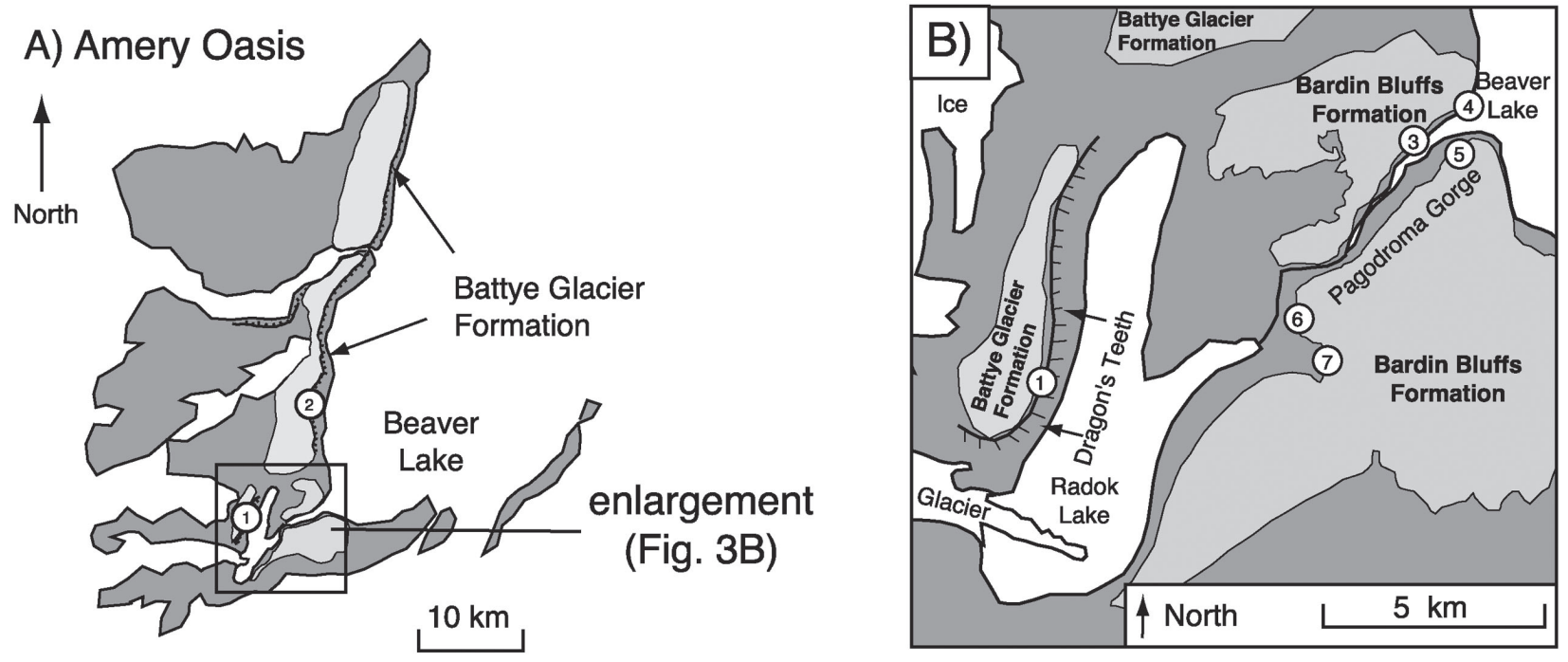

Other Bardin Bluffs Formation samples
(5) SE Pagodroma
(6) Bainmedart Top Bainmedart Bottom
(7) Glossopteris Top Glossopteris Bottom

C) Battye Glacier Formation
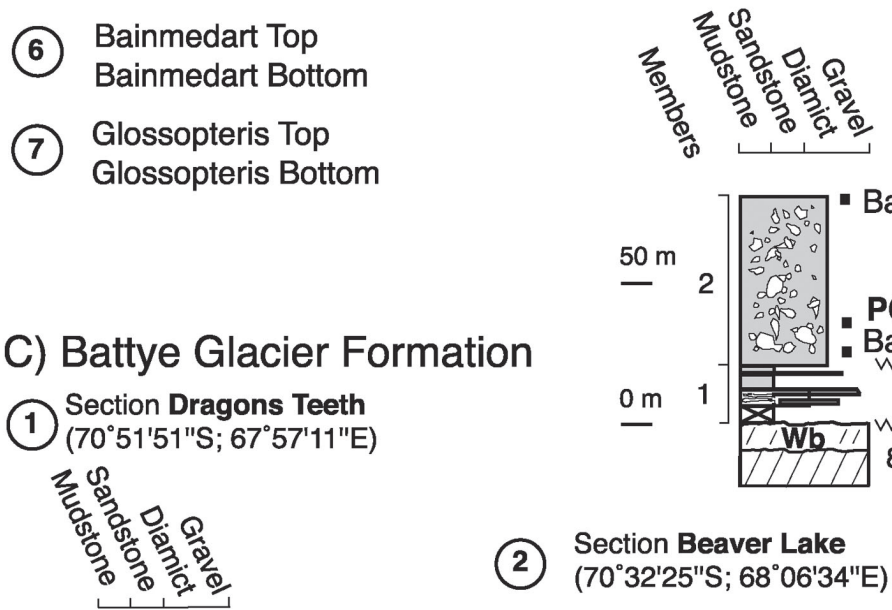

(3) Section Bardin Bluffs
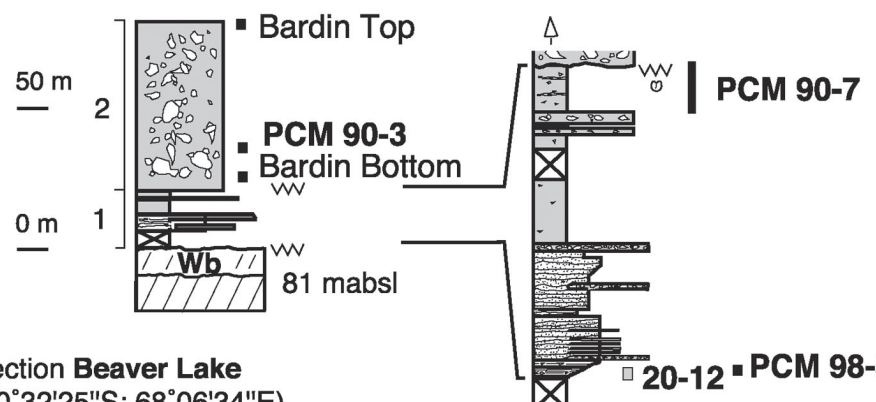
$\left(70^{\circ} 32^{\prime} 25^{\prime \prime} \mathrm{S} ; 68^{\circ} 06^{\prime} 34^{\prime \prime} \mathrm{E}\right)$
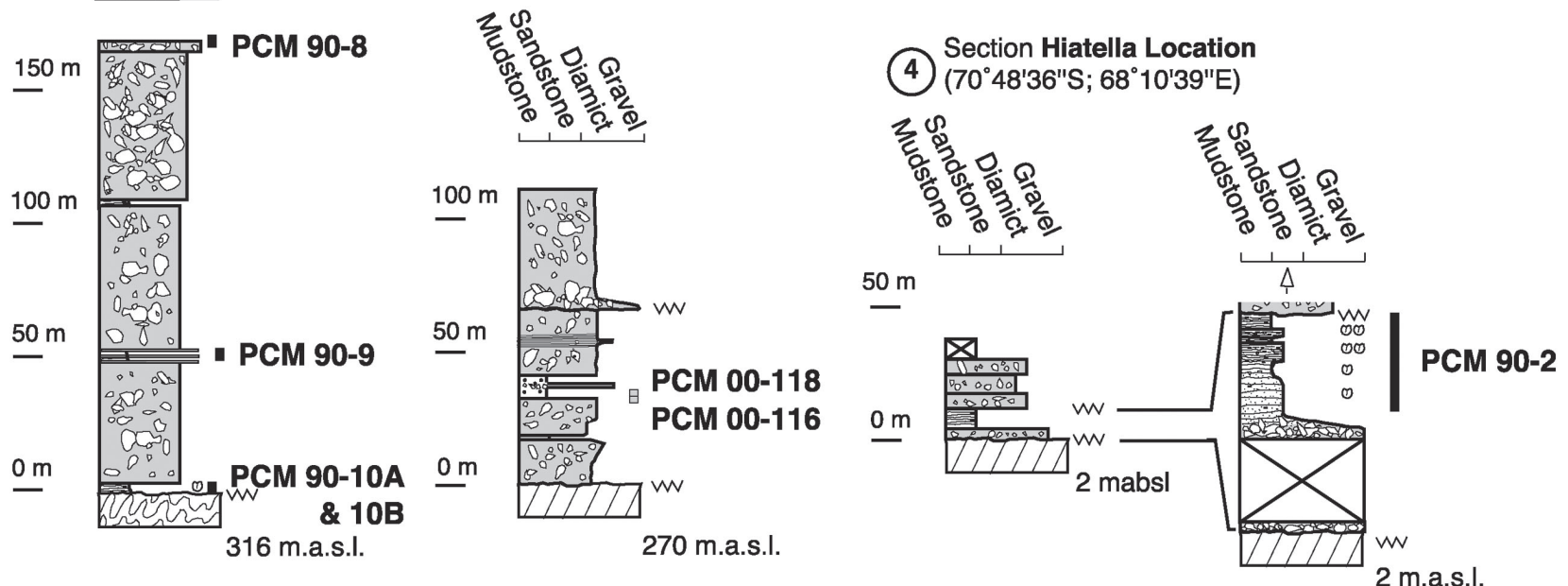

Figure 3. (a) Distribution of the Battye Glacier Formation and (b) Bardin Bluffs Formation at Amery Oasis. (c) Battye Glacier Formation sections and sample positions. (d) Bardin Bluffs Formation sections and sample positions. Section 1 is from Hambrey and McKelvey (2000a) and section 2 is from Whitehead et al. (2003) and sections 3 and 4 from Whitehead and McKelvey (2001). Symbols as in Figure 2. 


\section{Member 1}

In the Member 1 type section at least two mudstone intervals are diatom-bearing (Figure $3 \mathrm{~d}$ Section 3). Sample PCM 98-3Aa-026 contains numerous benthic diatoms that comprise $\sim 98 \%$ of the assemblage. These belong to the genera Amphora, Diploneis, Fragilaria, Hyalodiscus, Melosira, Navicula, Pinnularia and Trachyneis. The remainder of the assemblage are planktic species. Biogenic silica analysis of sample PCM 98-3Aa-026 indicates $\sim 2 \%$ opal (Table 1). A similar diatom assemblage was recorded from this same section by Bardin and Belevich (1985 sample 20-12). However, they identified planktic taxa such as Actinocyclus actinochilus (Ehrenberg) Simonsen (reported by them as Charcotia actinochilus) and Thalassiosira antarctica Comber, which are not observed in PCM 98-3Aa-026. The stratigraphically higher mudstone interval (sample PCM 90-7: Figure 3d Section 3) contains $\sim 1 \%$ opal. This diatom assemblage is of biostratigraphically mixed ages, as shown by the co-occurrence of A. actinochilus (3.1 Ma: Holocene) and Fragilariopsis praeinterfrigidaria (McCollum) Gersonde \& Bárcena (5.8-3.6 Ma). The assemblage is almost exclusively of planktic taxa, with the exception of one benthic specimen of Cocconeis pinnata Gregory. Diatoms were also recovered from Member 1 mudstone (sample PCM 90-2) at the "Hiatella location" (Figure 3d Section 4). This assemblage consists largely of extant planktic diatoms, with the exception of extinct $A$. ingens and Rouxia isopolica Schrader (Table 1). Some diatoms observed in sample PCM 90-2 occur in diatom-rich mudstone clasts (Appendix $2^{*}$ ). These clasts contain a higher abundance of diatoms than the surrounding mud matrix. The overall biogenic silica concentration of sample PCM $90-2$ is $\sim 2 \%$ opal.

\section{Member 2}

Many of the diamicts in Member 2 of the Bardin Bluffs Formation contain diatoms. The diatoms are predominantly planktic marine taxa. Mixing of diatoms of different ages was noted in five samples, e.g. "Bardin Top" (Figure 3d Section 3), and "Glossopteris Top," "Glossopteris Bottom," "Pagodroma SE," and "Bainmedart Top" (Figure 3b). These different-aged diatoms included Pliocene-Quaternary Fragilariopsis kerguelensis ( $\mathrm{O}^{\prime}$ Meara) Hasle, Pliocene F. praeinterfrigidaria, Miocene-Pliocene Denticulopsis hustedii (Simonsen \& Kanaya) Simonsen, Miocene Denticulopsis dimorpha (Schrader) Simonsen, Denticulopsis praedimorpha Barron ex Akiba and Eocene-Oligocene Pyxilla sp. fragment (Table 1). In sample "Glossopteris Top," centric diatoms and a silicoflagellate contain diatomaceous sediment within their valves and intraskeletal spaces, respectively. In addition, some diatoms occur as diatom-rich clasts, which are more biogenic than the host mud-matrix. The diamict assemblages also include sparse planktic freshwater diatoms of the genera Cyclotella, Stephanodiscus and Aulacoseira, a specimen of Tabellaria fenestrata (Lyngb.) Kützing, and a freshwater diatomaceous clast was recovered from sample "Bainmedart Bottom" (Harper \& Harwood 1992).

\section{Discussion}

The following discussion contains two sections. The first section, Diatom taphonomy, reviews the diatom data in order to identify the most likely mode of microfossil emplacement within the Pagodroma Group. The second section, Diatom biostratigraphy, discusses the assemblage composition in a biostratigraphic context, in order to provide age control for the individual formations of the Pagodroma Group.

\section{Diatom taphonomy}

Controversy surrounds the origins of diatoms found in outcrops of Cenozoic sedimentary strata in Antarctica (Miller \& Mabin 1998). This controversy has centered on the interpretation of diatoms within diamicts of the Sirius Group in

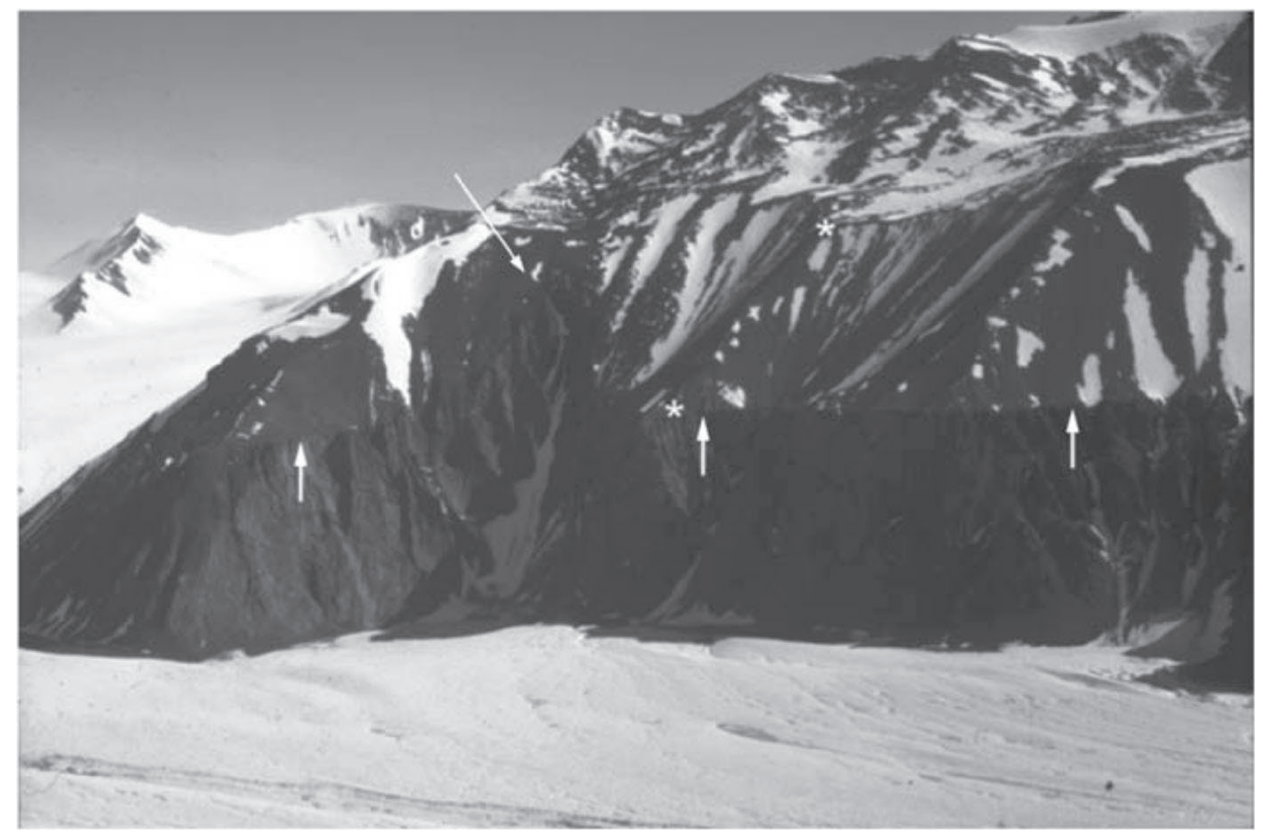

Figure 4. Southeastern face of Fisher Massif. View looking west. More than $320 \mathrm{~m}$ of Miocene Fisher Bench Formation overlying at $270 \mathrm{~m}$ above sea-level Proterozoic metavolcanic basement. In the background (center-right) the Cenozoic strata abut the same basement. The planar unconformity surface (small arrows) is a palaeofjord floor. However, in the most western part of the exposure (long arrow), the palaeofjord floor has become inclined and rises steeply as the palaeofjord wall. Asterisks indicate the top and bottom of the Fisher Bench Formation type section (see Figure 2c). 
the Transantarctic Mountains. Various processes have been proposed to explain these occurrences. Initially the diatoms were considered to be glacially reworked from now subglacial marine basins (Webb et al. 1983; Harwood 1986a,b; Scherer 1989, 1991; Harwood \& Webb 1998; Scherer et al. 1998; Tulaczyk et al. 1998). However, a counterargument is the contamination of outcrops by airborne diatoms. Wind erosion of diatom-bearing marine strata, and diatoms blown from marine and lake environments, are the alternative microfossil sources suggested (Burckle et al. 1988, 1997; Barrett 1996; Kellogg \& Kellogg 1996; Stroeven et al. 1996; Barrett et al. 1997). Furthermore, such aeolian processes may also deposit reworked diatoms onto the Antarctic ice sheet, to be eventually incorporated into later glacial deposits (Burckle et al. 1997). Another mechanism suggested for airborne diatom contamination is the Eltanin asteroid impact event in the Southern Ocean at ca 2.15 Ma (Gersonde et al. 1997). This asteroid impacted Cenozoic marine strata spanning the last 50 million years, and the consequent fallout could well have spread diatom-bearing ejecta widely over Antarctica. However, definite in situ biogenic marine deposition is now established for the Sørsdal Formation in the Vestfold Hills, some $400 \mathrm{~km}$ northeast of the Amery Oasis (Harwood et al. 2000; Whitehead et al. 2001). In view of this controversy and to help understand the mechanism for microfossil emplacement in the Pagodroma Group, its diatom assemblages and fossil size data have also been compared to similar data from Southern Ocean islands air samples (Chalmers et al. 1996), Antarctic ice cores (Burckle et al. 1988; Kellogg \& Kellogg 1996) and the Sirius Group (Harwood \& Webb 1998) (see below). We consider that in situ marine deposition and glacial reworking of older glacigene strata best explains diatom emplacement in the Pagodroma Group.

\section{Glacial Reworking}

Glacial reworking clearly is a mode of emplacement for some of the Pagodroma Group diatoms. For example, biostratigraphic and ecological mixing of freshwater and marine species, along with poor preservation and the occurrence of diatomaceous clasts in the Bardin Bluffs Formation, point to diatoms sourced by glacial reworking of older sediment. Similarly, reworked shell fragments in diamict (Bardin \& Kolosova 1988; Adamson \& Darragh 1991) and within sediment clasts in diamict (Stilwell et al. 2002) identify glacially reworked molluscs in the Pagodroma Group.

\section{In Situ Marine Deposition}

Marine mollusc fossils in the Battye Glacier and Bardin Bluffs Formations provide indisputable evidence for past marine deposition in the Lambert Graben. The mollusc valves generally occur in mudstones. Some valves are still articulated and in growth position (Whitehead et al. 2003). Others occur as scattered valves, sometimes crushed, in thinly laminated mudstone (Bardin \& Chepaljiga 1989). The molluscs are clearly in situ and their co-occurrence or close stratigraphic proximity to diatom-bearing samples strongly supports in situ marine deposition of these latter microfossils. Examples of such are the Battye Glacier Formation diatom-rich samples PCM 00-116, and PCM 00-118 (Whitehead et al. 2003), the Bardin Bluffs Formation samples PCM 983Aa-26 and 20-12 (Table 3). The Battye Glacier Formation sample PCM 00-118 contains up to $12 \%$ opal (Whitehead et al. 2003) and thus is comparable to sea-floor sediments from many open-marine areas on the present Antarctic continental shelf (Dunbar et al. 1989; Harris et al. 1997). However, most of the samples assessed for biogenic silica have $<3 \%$ opal, which is near or below the detection limit of the Mortlock and Froelich (1989) method used in the present study.

\section{Aeolian Contamination}

We have evaluated the potential for an aeolian origin of the Pagodroma Group diatom assemblages by comparing them with aeolian assemblages found in Antarctic continental ice cores ( $<20000$ years old). These ice-core diatoms have been blown onto the Antarctic ice sheets, and are predominately small freshwater and benthic marine taxa (Burckle et al. 1988; Kellogg \& Kellogg 1996; Harwood \& Webb 1998). At least $50 \%$ of the South Pole ice-core diatoms are benthic marine/ brackish and freshwater taxa (Kellogg \& Kellogg 1996). This aeolian assemblage contrasts with the predominantly marine planktic assemblages in most of the Pagodroma Group samples (Tables 1, 3).

Chemical and isotopic analyses of dust particles $(<25 \mu \mathrm{m})$ in Antarctic ice cores have identified South America as the principal source of aeolian material deposited in Antarctica (Gaudichet et al. 1986, 1988; Grousset et al. 1992; Delmas \& Petit 1994; Basile et al. 1997). The northern migration of the Antarctic Polar Front around the southern tip of South America causes the entrainment of dust by storms, which transport it to Antarctica (Burckle et al. 1988). This would suggest that the majority of aeolian diatoms delivered to Antarctica have also come from South America (Harwood $\&$ Webb 1998). The South Pole ice-core diatoms have been identified as predominately endemic Antarctic marine taxa (Kellogg \& Kellogg 1996), but most of the species are also compatible with a South American lake and adjacent marine biogeography (Hallegraeff 1986; Armand 1998; Kellogg \& Kellogg 2002).

Local Antarctic aeolian sources can adequately account for the small number of extant endemic Southern Ocean species recovered from ice cores. These include Fragilariopsis curta (Van Heurck) Hasle, F. kerguelensis, Fragilariopsis obliquecostata (Van Heurck) Heiden in Heiden \& Kolbe, and Fragilariopsis sublinearis (Van Heurck) Heiden (Burckle et al. 1988; Kellogg \& Kellogg 1996). The diatoms have been either reworked from Antarctic sediment exposures, released from the ocean surface (Kellogg \& Kellogg 1996), or else ablated from sea ice.

Aeolian-sourced extinct marine taxa in the Antarctic ice cores, although rare, include the cosmopolitan species $A$. ingens, Actinoptycus senarius Ehrenberg, D. hustedtii and Stephanopyxis turris (Greville \& Arnott) Ralfs in Pritchard, and the Southern Ocean endemic taxa Thalassiosira insigna (Jousé) Harwood \& Maruyama, Thalassiosira torokina Brady and Thalassiosira vulnifica (Gombos) Fenner (Burckle et al. 1988; Kellogg \& Kellogg 1996). Scarce extinct freshwater taxa recovered from Bardin Bluffs Formation and Fisher Bench For- 
Table 1. Siliceous microfossil data from the Pagodroma Group (present study) and from previous studies (Bardin \& Belevich 1985; Laiba \& Pushina 1997; Whitehead et al. 2003).

\begin{tabular}{|c|c|c|c|c|c|c|c|c|c|c|c|c|c|c|}
\hline \multirow{2}{*}{$\begin{array}{l}\text { Formations } \\
\text { Sample location }\end{array}$} & \multicolumn{11}{|c|}{ Bardin Bluffs } & \multicolumn{3}{|c|}{ Battye Glacier } \\
\hline & 1 & 1 & 5 & 5 & 3 & 4 & 4 & 2 & 1 & 1 & 1 & 6 & 6 & 6 \\
\hline Sample name & Bardin & Bardin & Gloss. & Gloss. & Pago & Bain. & Bain. & PCM & PCM & PCM & PCM & PCM & PCM & PCM \\
\hline Sample position/number & top & bottom & top & bottom & SE & top & bottom & $90-2$ & $90-3$ & $90-7$ & 98 & $90-8$ & $90-10 \mathrm{~A}$ & $90-10 \mathrm{~B}$ \\
\hline Lithology & $\mathrm{Dm}$ & $\mathrm{Dm}$ & $\mathrm{Dm}$ & $\mathrm{Dm}$ & $\mathrm{Dm}$ & $\mathrm{Dm}$ & $\mathrm{Dm}$ & Mud. & $\mathrm{Dm}$ & Mud. & Mud. & $\mathrm{Dm}$ & $\mathrm{Dm}$ & $\mathrm{Dm}$ \\
\hline Diatom abundance & 3 & 4 & 4 & 3 & 4 & 3 & 3 & 4 & 0 & 5 & 6 & 1 & 0 & 1 \\
\hline $\begin{array}{l}\text { Raw or concentrated slides } \\
\text { Biogenic silica (\% opal) }\end{array}$ & conc. & conc. & conc. & conc. & conc. & conc. & conc. & $\begin{array}{l}\text { conc. } \\
2 \%\end{array}$ & $\begin{array}{l}\text { conc. } \\
-\end{array}$ & $\begin{array}{l}\text { conc. } \\
1 \%\end{array}$ & $\begin{array}{l}\text { raw } \\
2 \%\end{array}$ & conc. & conc. & conc. \\
\hline
\end{tabular}

Diatoms: planktic marine

Actinocyclus actinochilus

Actinocyclus ingens

Actinocyclus ingens var. nodus

Actinocyclus aff. ingens

Actinocyclus karstenii

Actinocyclus octonarius

var. asteriscus

Actinoptycus senarius

Actinoptycus sp. A

Actinoptycus spp. (fragments)

Astromphalus spp. (fragments)

Azpeitia tabularis

Chaetoceros lorenzianus

Chaetoceros mitra

Chaetoceros subsecundus

Chaetoceros spp. (cyst)

Chaetoceros spp. (setae)

Chaetoceros sp. A (setae)

Coscinodiscus oculus-iridis

Coscinodiscus oculoides

Coscinodiscus marginatus

Coscinodiscus radiatus

Coscinodiscus sp. A

Coscinodiscus spp

(whole and frags.)

Dactyliosen antarcticus

Denticulopsis delicata

Denticulopsis dimorpha

Denticulopsis hustedtii

Denticulopsis ovata

Denticulopsis praedimorpha

Denticulopsis simonsenii

Denticulopsis vulgaris

Denticulopsis spp. (fragments)

Eucampia antarctica

Eunotia sp.

Fragilariopsis curta

Fragilariopsis interfrigidaria

Fragilariopsis kerguelensis

Fragiliariopsis praeinterfrigidaria

Fragiliariopsis sublinearis

Fragilariopsis spp. (fragments)

Nitzschia cholonii

Nitzschia delicatissima

Nitzschia frustulum

Nitzschia sp. A

Nitzschia reinholdii

Nitzschia sp.

Odontella weissflogii 


\begin{tabular}{|c|c|c|c|c|c|c|c|c|c|c|c|c|c|c|c|c|}
\hline \multirow[b]{3}{*}{6} & \multicolumn{6}{|c|}{ Fisher Bench } & \multicolumn{6}{|c|}{ Mt Johnston } & \multicolumn{4}{|c|}{ Previous studies } \\
\hline & & & & & & & & & & & & & \multirow{2}{*}{$\begin{array}{l}\text { Bardin } \\
1\end{array}$} & \multicolumn{2}{|l|}{ Battye } & \multirow{2}{*}{$\begin{array}{l}\text { Fisher } \\
9\end{array}$} \\
\hline & 9 & 9 & 9 & 9 & 8 & 7 & 10 & 10 & 10 & 10 & 10 & 10 & & 11 & 11 & \\
\hline $\mathrm{AM}$ & PCM & PCM & PCM & $\mathrm{AM}$ & $\mathrm{AM}$ & $\mathrm{AM}$ & PCM & PCM & $\mathrm{AM}$ & $\mathrm{AM}$ & $\mathrm{AM}$ & $\mathrm{AM}$ & & PCM & PCM & PCM \\
\hline 028 & $90-6$ & $90-11$ & $90-13$ & 015 & 017 & 026 & $90-4$ & $90-5$ & 002 & 003 & 006 & 014 & $20-12$ & $00-116$ & $00-118$ & 3672 \\
\hline $\mathrm{Dm}$ & Mud. & $\mathrm{Dm}$ & $\mathrm{Dm}$ & Mud. & $\mathrm{Dm}$ & $\mathrm{Dm}$ & $\mathrm{Dm}$ & $\mathrm{Dm}$ & $\mathrm{Dm}$ & $\mathrm{Dm}$ & $\mathrm{Dm}$ & $\mathrm{Dm}$ & Mud. & Mud. & Mud. & Mud. \\
\hline 0 & 1 & 4 & 0 & 6 & 4 & 1 & 1 & 1 & 0 & 1 & 2 & 1 & 6 & 6 & 6 & 5 \\
\hline conc. & conc. & conc. & conc. & raw & conc. & conc. & conc. & conc. & conc. & conc. & conc. & conc. & raw & raw & raw & conc. \\
\hline- & $1 \%$ & $1 \%$ & - & $\sim 2 \%$ & - & $1 \%$ & - & - & - & - & - & - & - & $7 \%$ & $12 \%$ & - \\
\hline
\end{tabular}

$\begin{array}{cc}\mathrm{R} & \mathrm{R} \\ - & - \\ - & - \\ \mathrm{X} & - \\ \mathrm{R} & \mathrm{R}\end{array}$

$\begin{array}{rl} & X \\ -5 & X\end{array}$

1-5

1-5

$\begin{array}{lll}- & R & R \\ - & R & R \\ - & - & - \\ - & C & R\end{array}$

1-5

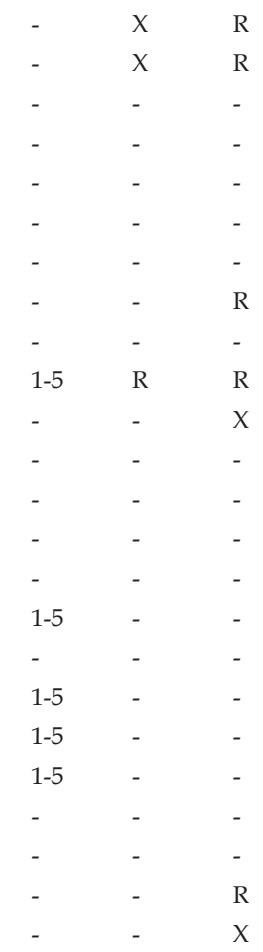


Table 1. continued.

\begin{tabular}{|c|c|c|c|c|c|c|c|c|c|c|c|c|c|c|}
\hline \multirow{2}{*}{$\begin{array}{l}\text { Formations } \\
\text { Sample location }\end{array}$} & \multicolumn{11}{|c|}{ Bardin Bluffs } & \multicolumn{3}{|c|}{ Battye Glacier } \\
\hline & 1 & 1 & 5 & 5 & 3 & 4 & 4 & 2 & 1 & 1 & 1 & 6 & 6 & 6 \\
\hline Sample name & Bardin & Bardin & Gloss. & Gloss. & Pago & Bain. & Bain. & PCM & PCM & PCM & PCM & PCM & PCM & PCM \\
\hline Sample position/number & top & bottom & top & bottom & SE & top & bottom & $90-2$ & $90-3$ & $90-7$ & 98 & $90-8$ & $90-10 \mathrm{~A}$ & $90-10 \mathrm{~B}$ \\
\hline Lithology & $\mathrm{Dm}$ & $\mathrm{Dm}$ & $\mathrm{Dm}$ & $\mathrm{Dm}$ & $\mathrm{Dm}$ & $\mathrm{Dm}$ & $\mathrm{Dm}$ & Mud. & $\mathrm{Dm}$ & Mud. & Mud. & $\mathrm{Dm}$ & $\mathrm{Dm}$ & $\mathrm{Dm}$ \\
\hline Diatom abundance & 3 & 4 & 4 & 3 & 4 & 3 & 3 & 4 & 0 & 5 & 6 & 1 & 0 & 1 \\
\hline Raw or concentrated slides & conc. & conc. & conc. & conc. & conc. & conc. & conc. & conc. & conc. & conc. & raw & conc. & conc. & conc. \\
\hline Biogenic silica (\% opal) & - & - & - & - & - & - & - & $2 \%$ & - & $1 \%$ & $2 \%$ & - & - & - \\
\hline
\end{tabular}

\section{Paralia sulcata}

Paralia sp.

Proboscia barboi

Pyxilla sp.

Rhizoslenia hebetata group

Rhizosolenia styliformis group

Rhizosolenia spp. (fragments)

Rouxia heteropolara

Rouxia isopolica

Rouxia naviculoides

Rouxia spp. (fragments)

Stellarima microtrias

Stellarima stellaris

Stellarima spp.

Stephanopyxis grunowii

Stephanopyxis splendidus

Stephanopyxis turris

Stephanopyxis $\mathrm{sp}$

Synedropsis sp.

Thalassiothrix sp. (fragments)

Thalassiothrix /

Thalassionema (frag.)

Thalassionema nitzschioides

Thalassionema nitzschioides

var. parva

Thalassiosira Antarctica

Thalassiosira gracils var. gracilis

Thalassiosira insigna

Thalassiosira inura

Thalassiosira nativa

Thalassiosira oliverana

Thalassiosira oestrupii

Thalassiosira sp.

(concentric areolae)

Thalassiosira spp. (fragments)

Diatoms: benthic marine

Achnanthes brevipes

Amphora spp.

Anaulus sp.

Caloneis sp.

Cocconeis sp A.

Cocconeis costata

Cocconeis fasciolata

Diploneis frickei

Diploneis oculata?

Diploneis subovalis

Diploneis smithii?

Diploneis bomboides

Diplonies and Navicula sp. (fragments) 


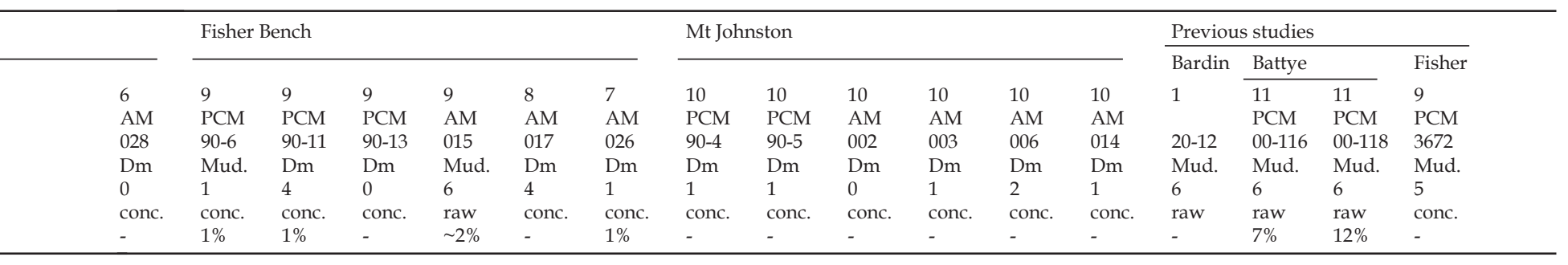


Table 1. continued.

\begin{tabular}{|c|c|c|c|c|c|c|c|c|c|c|c|c|c|c|}
\hline \multirow{2}{*}{$\begin{array}{l}\text { Formations } \\
\text { Sample location } \\
\text { Sample name } \\
\text { Sample position/number } \\
\text { Lithology } \\
\text { Diatom abundance } \\
\text { Raw or concentrated slides } \\
\text { Biogenic silica (\% opal) }\end{array}$} & \multicolumn{11}{|c|}{ Bardin Bluffs } & \multicolumn{3}{|c|}{ Battye Glacier } \\
\hline & $\begin{array}{l}1 \\
\text { Bardin } \\
\text { top } \\
\mathrm{Dm} \\
3 \\
\text { conc. } \\
-\end{array}$ & $\begin{array}{l}1 \\
\text { Bardin } \\
\text { bottom } \\
\text { Dm } \\
4 \\
\text { conc. } \\
-\end{array}$ & $\begin{array}{l}5 \\
\text { Gloss. } \\
\text { top } \\
\text { Dm } \\
4 \\
\text { conc. } \\
-\end{array}$ & $\begin{array}{l}5 \\
\text { Gloss. } \\
\text { bottom } \\
\text { Dm } \\
3 \\
\text { conc. } \\
-\end{array}$ & $\begin{array}{l}3 \\
\text { Pago } \\
\mathrm{SE} \\
\mathrm{Dm} \\
4 \\
\text { conc. } \\
- \\
\end{array}$ & $\begin{array}{l}4 \\
\text { Bain. } \\
\text { top } \\
\text { Dm } \\
3 \\
\text { conc. } \\
-\end{array}$ & $\begin{array}{l}4 \\
\text { Bain. } \\
\text { bottom } \\
\text { Dm } \\
3 \\
\text { conc. } \\
-\end{array}$ & $\begin{array}{l}2 \\
\text { PCM } \\
90-2 \\
\text { Mud. } \\
4 \\
\text { conc. } \\
2 \%\end{array}$ & $\begin{array}{l}1 \\
\text { PCM } \\
90-3 \\
\text { Dm } \\
0 \\
\text { conc. } \\
-\end{array}$ & $\begin{array}{l}1 \\
\text { PCM } \\
90-7 \\
\text { Mud. } \\
5 \\
\text { conc. } \\
1 \%\end{array}$ & $\begin{array}{l}1 \\
\text { PCM } \\
98 \\
\text { Mud. } \\
6 \\
\text { raw } \\
2 \%\end{array}$ & $\begin{array}{l}6 \\
\text { PCM } \\
90-8 \\
\text { Dm } \\
1 \\
\text { conc. } \\
-\end{array}$ & $\begin{array}{l}6 \\
\mathrm{PCM} \\
90-10 \mathrm{~A} \\
\mathrm{Dm} \\
0 \\
\text { conc. } \\
-\end{array}$ & $\begin{array}{l}6 \\
\text { PCM } \\
90-10 \mathrm{~B} \\
\mathrm{Dm} \\
1 \\
\text { conc. } \\
-\end{array}$ \\
\hline Fragilaria spp & - & - & - & - & - & - & - & - & - & - & 1 & - & - & - \\
\hline Grammatophora spp. & - & - & - & - & - & - & - & - & - & - & - & - & - & - \\
\hline Hyalodiscus valens & - & - & - & - & - & - & - & - & - & - & 12 & - & - & - \\
\hline$\underline{\text { Hyalodiscus obsoletus }}$ & - & - & - & - & - & - & - & - & - & - & - & - & - & - \\
\hline Hyalodiscus zonulatus & - & - & - & - & - & - & - & - & - & - & 14 & - & - & - \\
\hline Hyalodiscus sp. & - & - & - & - & - & - & - & - & - & - & - & - & - & - \\
\hline Isthmia sp. (fragments) & - & - & - & - & - & - & - & - & - & - & - & - & - & - \\
\hline Melosira sp. & - & - & - & - & - & - & - & - & - & - & 205 & - & - & - \\
\hline Podosira sp. & - & - & - & - & - & - & - & - & - & - & - & - & - & - \\
\hline Navicula cryophila & - & - & - & - & - & - & - & - & - & - & - & - & - & - \\
\hline Navicula directa & - & - & - & - & - & - & - & - & - & - & $\mathrm{r}$ & - & - & - \\
\hline Navicula glaciei & - & - & - & - & - & - & - & - & - & - & - & - & - & - \\
\hline Navicula retusa & - & - & - & - & - & - & - & - & - & - & - & - & - & - \\
\hline Navicula spp. & - & - & - & - & - & - & - & - & - & - & - & - & - & - \\
\hline Nitzschia acicularis & - & - & - & - & - & - & - & - & - & - & - & - & - & - \\
\hline$\underline{\text { Nitzschia scabra }}$ & - & - & - & - & - & - & - & - & - & - & - & - & - & - \\
\hline Porosira antarctica & - & - & - & - & - & - & - & - & - & - & - & - & - & - \\
\hline Porosira pseudeodenticulata & - & - & - & - & - & - & - & - & - & - & 1 & - & - & - \\
\hline Pinnularia quadratea & - & - & - & - & - & - & - & - & - & 19 & - & - & - & - \\
\hline Rhabdonema japonica group & - & - & - & - & - & - & - & - & - & - & - & - & - & - \\
\hline Rhabdonema sp.cf. R. elegans & - & - & - & - & - & - & - & - & - & - & - & - & - & - \\
\hline Rhabdonema spp. & - & - & - & - & - & - & - & - & - & - & - & - & - & - \\
\hline Rhaphoneis spp. & - & - & - & - & - & - & - & - & - & - & - & - & - & - \\
\hline Stictodiscus hardmanianus & - & - & - & - & - & - & - & - & - & - & - & - & - & - \\
\hline Synedra sp. & - & - & - & - & - & - & - & - & - & - & $\mathrm{r}$ & - & - & - \\
\hline Trachyneis aspera & - & - & - & - & - & - & - & - & - & 7 & - & - & - & - \\
\hline$\frac{\text { Triceratium oamaruense }}{\text { var. sparsipunctatum }}$ & - & - & - & - & - & - & - & - & - & - & - & - & - & - \\
\hline Trinacria excavata & - & - & - & - & - & - & - & - & - & - & - & - & - & - \\
\hline Trigonium arcticum & - & - & - & - & - & - & - & - & - & - & - & - & - & - \\
\hline $\begin{array}{l}\text { Gen. et sp. B } \\
\quad \text { (of Harwood (1986a)) }\end{array}$ & - & - & - & - & - & - & - & - & - & - & - & - & - & - \\
\hline
\end{tabular}

\section{Diatoms: benthic, possible non-marine}

Amphora sp. A

Cocconeis pinnata (non-marine?)

Diploneis sp. A

Gomphonema angustatum var. A

Gomphonema angustatum var. B

Tabellaria fenestrata

Tabellaria sp.

Aulacoseira sp.

Cyclotella sp.

Stephanodiscus spp. 


\begin{tabular}{|c|c|c|c|c|c|c|c|c|c|c|c|c|c|c|c|c|}
\hline \multirow[b]{3}{*}{6} & \multicolumn{6}{|c|}{ Fisher Bench } & \multicolumn{6}{|c|}{ Mt Johnston } & \multicolumn{4}{|c|}{ Previous studies } \\
\hline & & & & & & & & & & & & & \multirow{2}{*}{$\begin{array}{l}\text { Bardin } \\
1\end{array}$} & \multicolumn{2}{|l|}{ Battye } & \multirow{2}{*}{$\begin{array}{l}\text { Fisher } \\
9\end{array}$} \\
\hline & 9 & 9 & 9 & 9 & 8 & 7 & 10 & 10 & 10 & 10 & 10 & 10 & & 11 & 11 & \\
\hline 028 & $90-6$ & $90-11$ & $90-13$ & 015 & 017 & 026 & $90-4$ & $90-5$ & 002 & 003 & 006 & 014 & $20-12$ & $00-116$ & $00-118$ & 3672 \\
\hline $\mathrm{Dm}$ & Mud. & $\mathrm{Dm}$ & $\mathrm{Dm}$ & Mud. & $\mathrm{Dm}$ & $\mathrm{Dm}$ & $\mathrm{Dm}$ & $\mathrm{Dm}$ & $\mathrm{Dm}$ & $\mathrm{Dm}$ & $\mathrm{Dm}$ & $\mathrm{Dm}$ & Mud. & Mud. & Mud. & Mud. \\
\hline 0 & 1 & 4 & 0 & 6 & 4 & 1 & 1 & 1 & 0 & 1 & 2 & 1 & 6 & 6 & 6 & 5 \\
\hline conc. & conc. & conc. & conc. & raw & conc. & conc. & conc. & conc. & conc. & conc. & conc. & conc. & raw & raw & raw & conc. \\
\hline
\end{tabular}


Table 1. continued.

\begin{tabular}{|c|c|c|c|c|c|c|c|c|c|c|c|c|c|c|}
\hline \multirow{2}{*}{$\begin{array}{l}\text { Formations } \\
\text { Sample location } \\
\text { Sample name } \\
\text { Sample position/number } \\
\text { Lithology } \\
\text { Diatom abundance } \\
\text { Raw or concentrated slides } \\
\text { Biogenic silica (\% opal) }\end{array}$} & \multicolumn{11}{|c|}{ Bardin Bluffs } & \multicolumn{3}{|c|}{ Battye Glacier } \\
\hline & $\begin{array}{l}1 \\
\text { Bardin } \\
\text { top } \\
\text { Dm } \\
3 \\
\text { conc. } \\
-\end{array}$ & $\begin{array}{l}1 \\
\text { Bardin } \\
\text { bottom } \\
\text { Dm } \\
4 \\
\text { conc. } \\
-\end{array}$ & $\begin{array}{l}5 \\
\text { Gloss. } \\
\text { top } \\
\text { Dm } \\
4 \\
\text { conc. } \\
-\end{array}$ & $\begin{array}{l}5 \\
\text { Gloss. } \\
\text { bottom } \\
\text { Dm } \\
3 \\
\text { conc. } \\
-\end{array}$ & $\begin{array}{l}3 \\
\text { Pago } \\
\text { SE } \\
\text { Dm } \\
4 \\
\text { conc. } \\
-\end{array}$ & $\begin{array}{l}4 \\
\text { Bain. } \\
\text { top } \\
\text { Dm } \\
3 \\
\text { conc. } \\
-\end{array}$ & $\begin{array}{l}4 \\
\text { Bain. } \\
\text { bottom } \\
\text { Dm } \\
3 \\
\text { conc. } \\
-\end{array}$ & $\begin{array}{l}2 \\
\text { PCM } \\
90-2 \\
\text { Mud. } \\
4 \\
\text { conc. } \\
2 \%\end{array}$ & $\begin{array}{l}1 \\
\text { PCM } \\
90-3 \\
\text { Dm } \\
0 \\
\text { conc. } \\
-\end{array}$ & $\begin{array}{l}1 \\
\text { PCM } \\
90-7 \\
\text { Mud. } \\
5 \\
\text { conc. } \\
1 \%\end{array}$ & $\begin{array}{l}1 \\
\text { PCM } \\
98 \\
\text { Mud. } \\
6 \\
\text { raw } \\
2 \%\end{array}$ & $\begin{array}{l}6 \\
\text { PCM } \\
90-8 \\
\text { Dm } \\
1 \\
\text { conc. } \\
-\end{array}$ & $\begin{array}{l}6 \\
\text { PCM } \\
90-10 \mathrm{~A} \\
\mathrm{Dm} \\
0 \\
\text { conc. } \\
-\end{array}$ & $\begin{array}{l}6 \\
\text { PCM } \\
90-10 B \\
\text { Dm } \\
1 \\
\text { conc. } \\
-\end{array}$ \\
\hline \multicolumn{15}{|l|}{ Dinoflagellates } \\
\hline Actiniscus pentasteris & - & - & - & - & - & - & - & - & - & 5 & - & - & - & - \\
\hline \multicolumn{15}{|l|}{ Silicoflagellates } \\
\hline Distephanus spp. & - & - & 1 & - & 1 & - & - & 1 & - & 1 & - & - & - & - \\
\hline \multicolumn{15}{|l|}{ Ebridians } \\
\hline Pseudammodochium lingii & - & - & - & - & - & - & - & - & - & - & - & - & - & - \\
\hline Sponge spicules & 5 & 11 & 4 & 1 & 6 & 2 & - & - & - & 1 & $\mathrm{r}$ & - & - & 1 \\
\hline Radiolaria & - & - & 2 & 1 & 2 & 1 & 1 & - & - & - & - & - & - & - \\
\hline Chrysophytes & 3 & 5 & - & - & 1 & - & - & - & - & - & - & - & - & - \\
\hline
\end{tabular}

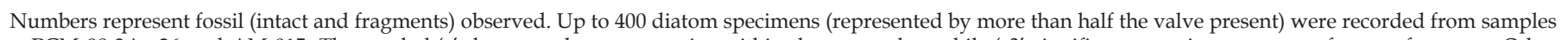
PCM 98-3Aa-26 and AM-015. The symbol ' $r$ ' denotes other rare species within these samples, while ' $r$ ?' signifies uncertain taxonomy of a rare fragment. Other abbreviations: Bardin, Bardin Bluffs; Gloss., Glossopteris; Pago., Pagodroma; Bain., Bainmedart; Battye, Battye Glacier; Fisher, Fisher Bench; Dm, diamict; Mud., mudstone; conc., concentrated. Underlined species are those only identified in previous studies. Previous Bardin Bluffs Formation sample data are number of specimens seen in the preparation and per traverse of the slide (/T). Abbreviations in previous Battye Glacier Formation sample data are: $\mathrm{C},>1$ valve per field of view; F, >1 valve per 10 fields of view; R, 3 valves in the preparation; X, 1 valve in the preparation. Abbreviations in previous Fisher Bench Formation data are: VR, 1 valve in the preparation; R, 1-10 valves in the preparation; F, 1-3 per traverse of slide. opal, biogenic silica. Some data from Whitehead et al. (2003) have been omitted because they do not help to further constrain the biostratigraphic age.

mation samples (e.g. "Bainmedart Bottom" and PCM 90-11) may also be aeolian contaminants. However, exposures of diatomaceous strata suitable to source (for aeolian transport) the several extinct species of diverse ages found in the Pagodroma Group are not yet known in Antarctica; neither do the Pagodroma Group diatom assemblages resemble the aeolian assemblages found in ice cores. In particular, there is no appreciable component of freshwater taxa (Table 3). For these two reasons we consider any aeolian contribution to the Pagodroma Group assemblages not to have been a major one. Furthermore many of the diatomaceous clasts in the Pagodroma Group, and in particular those in the Bardin Bluffs Formation sample PCM 90-2, exceed the particle size of continental loess, and of aeolian dust and diatom material recovered from Antarctic ice cores (Figure 9). Thus such clasts are unlikely to be of aeolian origin.

\section{Asteroid Impact Fallout}

It has been suggested the Eltanin asteroid impact in the Southern Ocean at $c a 2.15 \mathrm{Ma}$ caused the ejection of diatomaceous marine sediment spanning the last 50 million years, and through fallout this material may have been widely redeposited over Antarctica (Gersonde et al. 1997). Presumably such fallout would be biostratigraphically mixed, containing planktic marine diatoms spanning the interval between 50 and 2.15 Ma. Samples with biostratigraphically mixed planktic marine species do occur in the Bardin Bluffs Forma- tion (Table 3; Figure 8), but their ages are generally <14.2 Ma and do not span the interval 50-2.15 Ma. However, sample "Pagodroma SE" (of the Bardin Bluffs Formation) produced a single fragment of Pyxilla sp., very probably the Southern Ocean species Pyxilla reticulata Grove \& Sturt, with a last occurrence datum of $30.1 \mathrm{Ma}$ (Scherer et al. 2000). Because the time interval between 30.1 and $14.2 \mathrm{Ma}$ is not represented, inheritance through glacial reworking rather than by impact fallout appears to be the more likely interpretation of this fossil. Biostratigraphic mixing of Miocene taxa is apparent in Fisher Bench Formation sample AM-015, where 14.5-12.5 Ma taxa are admixed with 11.7-8.5 Ma taxa (Figure 7) (discussed below). In the absence of both $>14.5 \mathrm{Ma}$ and $<8.5 \mathrm{Ma}$ diagnostic taxa, glacial reworking would again appear to be a simpler interpretation.

\section{Summary}

In the light of the evidence reviewed above we consider the diatom assemblages of the Pagodroma Group to predominantly reflect (i) in situ marine deposition and/or (ii) glaciomarine reworking of older Cenozoic strata. In consequence both the non-mixed and mixed biostratigraphic assemblages encountered are to be expected. In arriving at these two conclusions concerning diatom emplacement, we are most strongly influenced by (i) the presence of in situ macrofossils in the Battye Glacier Formation and of in situ macrofossils and foraminifers in the Bardin Bluffs Formation; (ii) the 
Fisher Bench

\begin{tabular}{|c|c|c|c|c|c|c|c|c|c|}
\hline \multicolumn{6}{|c|}{ Mt Johnston } & \multicolumn{4}{|c|}{ Previous studies } \\
\hline & & & & & & \multirow{2}{*}{$\begin{array}{l}\text { Bardin } \\
1\end{array}$} & \multicolumn{2}{|l|}{ Battye } & \multirow{2}{*}{$\begin{array}{l}\text { Fisher } \\
9\end{array}$} \\
\hline 10 & 10 & 10 & 10 & 10 & 10 & & 11 & 11 & \\
\hline PCM & PCM & $\mathrm{AM}$ & $\mathrm{AM}$ & $\mathrm{AM}$ & $\mathrm{AM}$ & & PCM & PCM & PCM \\
\hline $90-4$ & $90-5$ & 002 & 003 & 006 & 014 & $20-12$ & $00-116$ & $00-118$ & 3672 \\
\hline $\mathrm{Dm}$ & $\mathrm{Dm}$ & $\mathrm{Dm}$ & $\mathrm{Dm}$ & $\mathrm{Dm}$ & $\mathrm{Dm}$ & Mud. & Mud. & Mud. & Mud. \\
\hline 1 & 1 & 0 & 1 & 2 & 1 & 6 & 6 & 6 & 5 \\
\hline conc. & conc. & conc. & conc. & conc. & conc. & raw & $\begin{array}{l}\text { raw } \\
7 \%\end{array}$ & $\begin{array}{l}\text { raw } \\
12 \%\end{array}$ & conc. \\
\hline
\end{tabular}

\begin{tabular}{lllllll}
6 & 9 & 9 & 9 & 9 & 8 & 7 \\
AM & PCM & PCM & PCM & AM & AM & AM \\
028 & $90-6$ & $90-11$ & $90-13$ & 015 & 017 & 026 \\
Dm & Mud. & Dm & Dm & Mud. & Dm & Dm \\
0 & 1 & 4 & 0 & 6 & 4 & 1 \\
conc. & conc. & conc. & conc. & raw & conc. & conc. \\
- & $1 \%$ & $1 \%$ & - & $\sim 2 \%$ & - & $1 \%$ \\
\hline
\end{tabular}

$-\quad-\quad 5$

5

$-$

$-$

$-$

$-\quad-$

$-$

r $\quad-$

- $\quad-$

$-$

$\begin{array}{cccccc}- & - & - & - & X & R \\ - & - & - & - & - & - \\ - & - & - & - & X & R\end{array}$

$5 \quad-\quad 12$

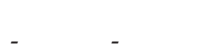

(n)

frequent absence of biostratigraphic admixing in many samples; (iii) the predominantly planktic composition of most Pagodroma Group diatom assemblages, in marked contrast to the marine planktic, benthic and lacustrine assemblages recovered from polar ice cores (Burckle et al. 1988; Kellogg \& Kellogg 1996; Harwood \& Webb 1998); and (iv) the absence of microfossil assemblages adequately spanning the 50$2.15 \mathrm{Ma}$ interval supposedly encompassed by the Eltanin asteroid fallout. Although some aeolian contamination is very possible, the apparent lack of stratigraphic sources appropriate to account for the Pagodroma Group assemblages, their compositional difference to aeolian assemblages in ice cores, and the as yet palaeontologically unproven status of the suggested Eltanin asteroid fallout contribution, we view any aeolian contribution as being only minor. The glaciomarine depositional scenario proposed for the Pagodroma Group by Hambrey and McKelvey (2000a, b) has been modified (Figure 10) to incorporate both in situ marine diatom deposition and glacial reworking processes.

\section{Diatom biostratigraphy}

Because the following biostratigraphic discussion assumes that glacial reworking processes may have been operative, the maximum age of most samples can be constrained only to the first occurrence datum of the youngest fossil present.

\section{Mt Johnston Formation}

The presence (Table 1) of Stephanopyxis splendidus and Pyxilla sp. (probably $P$. reticulata) suggests, in the first instance, marine deposition at some time in the Early Oligocene, between $\mathrm{ca} 35$ and $33 \mathrm{Ma}$ (Figure 7). However, it is unclear if these Mt Johnston Formation diatoms are in situ or are glacially reworked into diamicts (Figure 10). The fact that there is no biostratigraphic mixing with assemblages found in the nearby Middle to Upper Miocene Fisher Bench Formation (discussed below) does restrict deposition of the Mt Johnston Formation to an interval between Early Oligocene (ca $35 \mathrm{Ma}$ ) and the Middle to Late Miocene (>12.1 Ma).

\section{Fisher Bench Formation}

Of the six samples from this formation (Figure 2c; Table 1), only sample PCM 90-13 is barren of diatoms. The microfossils are most abundant in the two mudstone samples AM 015 and 36721-4, the latter sample that of Laiba and Pushina (1997). The relatively high abundance of diatoms in these restricted intervals of fine sediment, and the rarity of diatoms in the associated diamict facies, strongly suggests the mudstone microfossils to be in situ.

Diatoms in the basal sample AM-015 suggest deposition in the Middle to Late Miocene. An age between 14.2 and $12.5 \mathrm{Ma}$ was assigned for this sample in McKelvey et al. (2001), based on the presence of Actinocyclus ingens var. nodus Baldauf (first occurrence $14.5 \mathrm{Ma}$ and last occurrence $12.5 \mathrm{Ma}$ ) and of $D$. simonsenii, with a first occurrence of 14.2 Ma (now considered to be 14.3 Ma). However, it is possible to further restrict the age to between 11.7 and $8.5 \mathrm{Ma}$, based on additional data (Figure $7 \mathrm{~b}$ ). This would necessitate interpreting the presence of $A$. ingens var. nodus (first occurrence $12.5 \mathrm{Ma}$ ) as a consequence of reworking of older strata. This younger age is supported by the presence of Denticulopsis ovata (Schrader) Yanagisawa \& Akiba, the range of which spans ca 12.11-8.5 Ma. Support for an age of <11.7 Ma for AM-015 comes from the presence of D. vulgaris. This species has a first occurrence of $13.0 \mathrm{Ma}$ in the northern Pacific (Yanagisawa \& Akiba 1998), but appears later in the Kerguelen Plateau ODP Site 1138A, with a first occurrence in the D. dimorpha Zone, i.e. between 11.7 and $10.7 \mathrm{Ma}$ (Bohaty et al. 
Figure 5. (1) Denticulopsis hustedtii (Glossopteris Top). $(2,3)$ Denticulopsis dimorpha (Bardin Top). (4) Denticulopsis ovata (Glossopteris Bottom). (5) Denticulopsis praedimorpha (Bardin Top). (6) Denticulopsis simonsenii (AM-015). (7) Denticulopsis delicata fragment (AM-015). (8) Fragilariopsis praeinterfrigidaria (Glossopteris Top). (9) Fragilariopsis interfrigidaria fragment (Glossopteris Bottom). (10) Nitzschia reinholdii fragment (PCM 90-7). (11) Fragilariopsis kerguelensis (Glossopteris Top). (12) Thalassionema nitzschioides (Glossopteris Bottom). (13) Pyxilla sp. probably Pyxilla reticulata fragment (AM006). (14) Gomphonema angustatum var. A (PCM 90-11). (15) Gomphonema angustatum var. B (PCM 90-11). (16) Rhabdonema japonica fragment (AM-015). 17 Rouxia isopolica (PCM 90-7). (18) Rouxia heteropolara (Bardin Bottom). 19 Rhaphoneis sp. (AM-015).

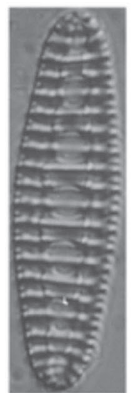

1

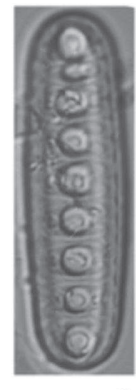

2
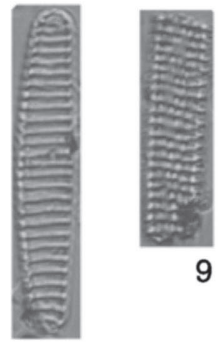

9

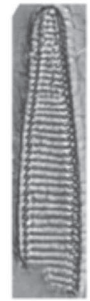

8

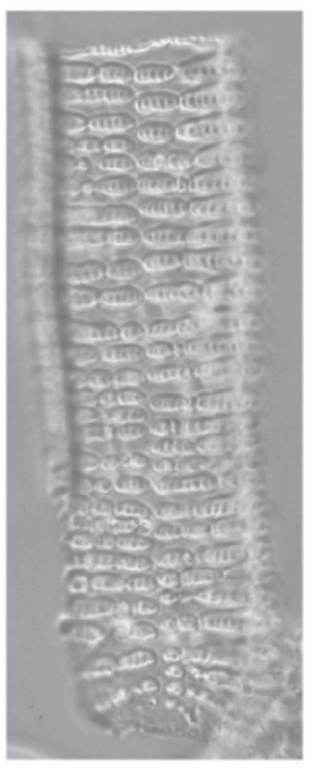

16

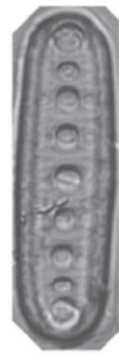

3
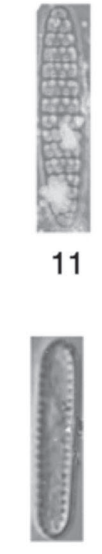

12

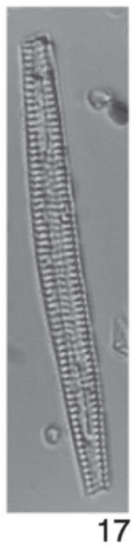

18

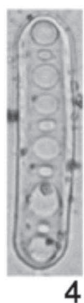

4

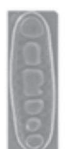

5
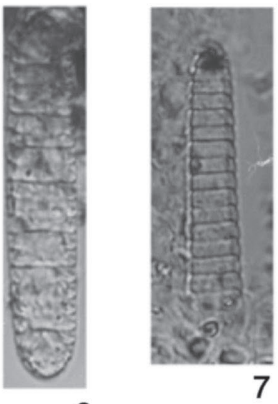

6

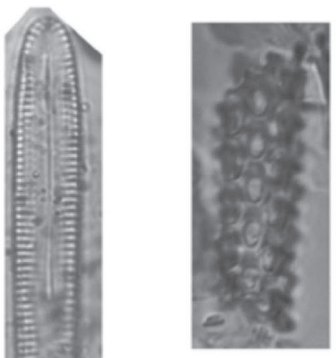

13

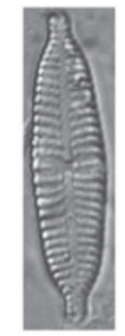

14

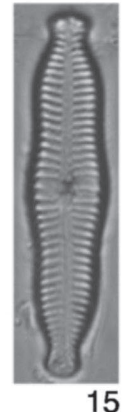

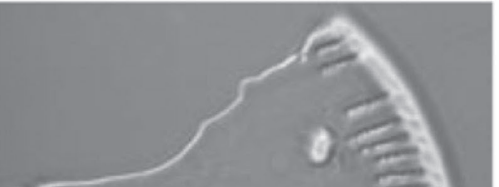

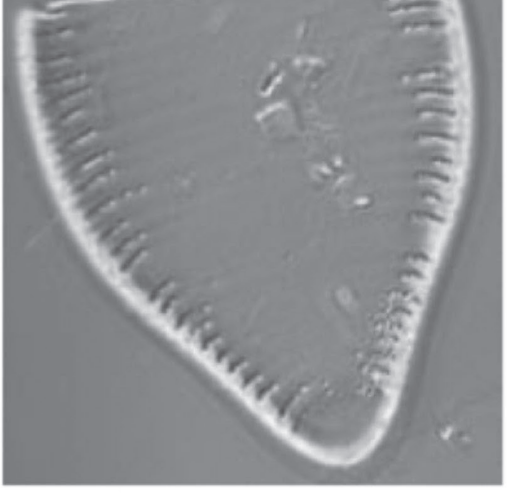

19

2003). The presence of Proboscia barboi (Brün) Jordan \& Priddle (first occurrence of $12.7 \mathrm{Ma}$ ) in AM-015, further supports an age $<12.7 \mathrm{Ma}$.

Sample 36721-4 (Figure 2c, Section 3) of Laiba and Pushina (1997) contains Miocene marine diatoms including D. simonsenii, which constrains the age only broadly to between 14.3 and 4.9 Ma. Sample 36721-4 is also rich in benthic diatoms, suggesting either deposition in a marine environment $<50 \mathrm{~m}$ deep or else sediment transport by syndepositional slumping of shallow-marine sediments from near the former fjord wall (Laiba \& Pushina 1997). Overall, the benthic species in the Fisher Bench Formation support a Neogene age. Sample PCM 90-6 (Figure 2c Section 2) contains a few intact and fragmented diatoms, which add no further age constraint. Rhabdonema japonica Tempére \& Brün, Gen. et sp. C Harwood (1986a) and Isthmia sp. occur in AM-015;
H. obsoletus, Rhabdonema sp. and Rhaphoneis spp. occur in 36721-4. Examination of the assemblages in Kellogg and Kel$\operatorname{logg}$ (2002) indicates that the above taxa are no longer found in the modern Antarctic environment, but they are present in shallow-marine Neogene sediments in the Ross Sea (Harwood 1986a,b; Harwood et al. 1989; Winter 1995; Barrett et al. 1998) and in Prydz Bay (Harwood et al. 2000; Whitehead et al. 2001).

As previously noted, the Fisher Bench Formation diamict sample PCM 90-11 contains two assemblages that contrast in preservation and ecology. The less abundant, but conspicuously well-preserved diatoms (often complete frustules) are the benthic species Gomphonema angustatum var. sp. A, G. angustatum var. sp. B, Diploneis sp. A and Amphora sp. A. Gomphonema angustatum is a freshwater species (Cramer 1979; Beaver 1981), as could also be the variants G. angustatum var. 
Figure 6. (1) Actinocyclus actinochilus (PCM 90-7). (2) Actinocyclus ingens (AM-015). (3,4) Actinocyclus ingens var. nodus (AM-015). (5) Actinocyclus karstenii (Bardin Top). (6) Eucampia antarctica (Bardin Top). (7) Coscinodiscus marginatus (Bardin Bottom). (8) Stellarima stellaris fragment, arrows indicate labiate arrangement and lack of central hyaline area (different to $S$. microtrias) (AM015). (9) Stellarima microtrias (AM-015).

(10) Stephanopyxis splendidus (arrows indicate distinctive processes) (AM-006). (11) Stephanopyxis turris (AM-006). (12) Thalassiosira oliverana (Glossopteris Bottom). (13) Radiolarian fragment containing diatomite (Glossopteris Bottom). (14) Diatomaceous aggregate (PCM 90-2). (15) Diatomaceous aggregate with an arrow indicating a fragment of Fragilariopsis kerguelensis (Bardin Bottom). (16) Silicoflagellate Distephanus speculum speculum "pseudofibula plexus" containing diatomite (Glossopteris Top). (17) Rhizosolenia hebetata fragment (PCM 907). (18) Stephanodiscus spp. fragment and arrow pointing at Aulacoseira sp. (Pagodroma SE). (19) Aulacoseira sp. (Bainmedart Bottom). (20) Coscinodiscus sp. A (AM-015). (21) Ebridian Pseudammodochium lingii (AM-015). (22) Trinacria excavata (AM-015). (23) Thalassiosira gracilis var. gracilis (Bainmedart Top). (24) Actinoptycus sp. A (PCM 90-7). (25) Actinoptycus senarius (PCM 90-7).
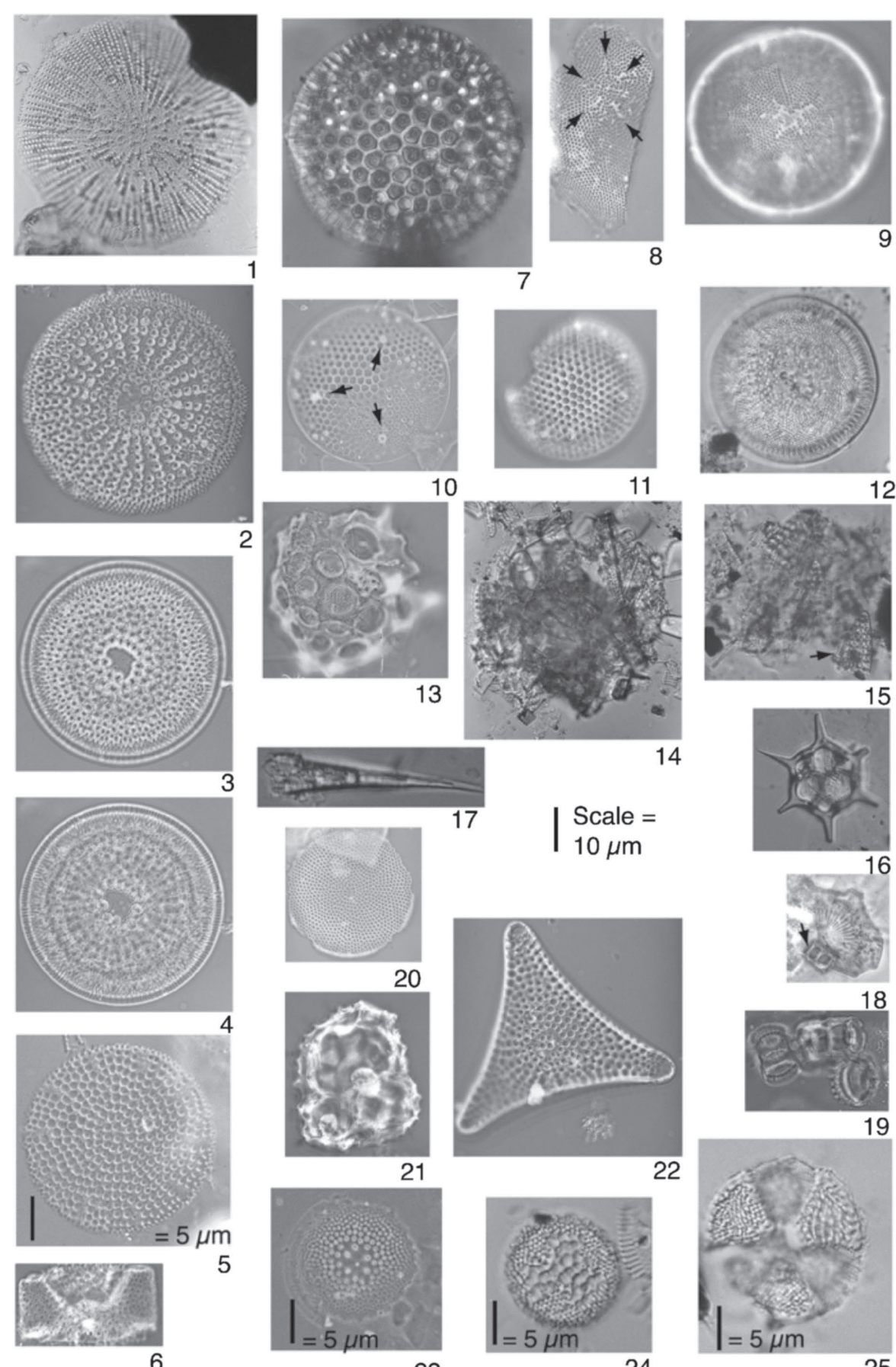

5

6

sp. A, and G. angustatum var. sp. B. While Amphora and Diploneis are typically marine genera, rare freshwater species do exist, and their association with G. angustatum var. spp. A and $B$ suggests that these Fisher Bench diatoms are non-marine species. They possibly originated from lakes discharging into the Lambert Graben during accumulation of Fisher Bench Formation diamicts.

The most abundant diatoms in sample PCM 90-11 are highly fragmented planktic marine taxa. Their fragmented nature suggests glacial reworking, because in situ marine deposition should allow for good diatom preservation. Later glacial overriding and sediment shearing can cause diatom breakage (Scherer et al. 2001); but the excellent preserva-
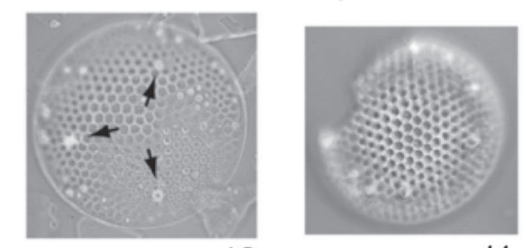

10

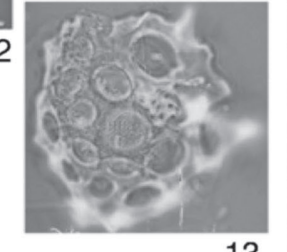

3

\section{.}
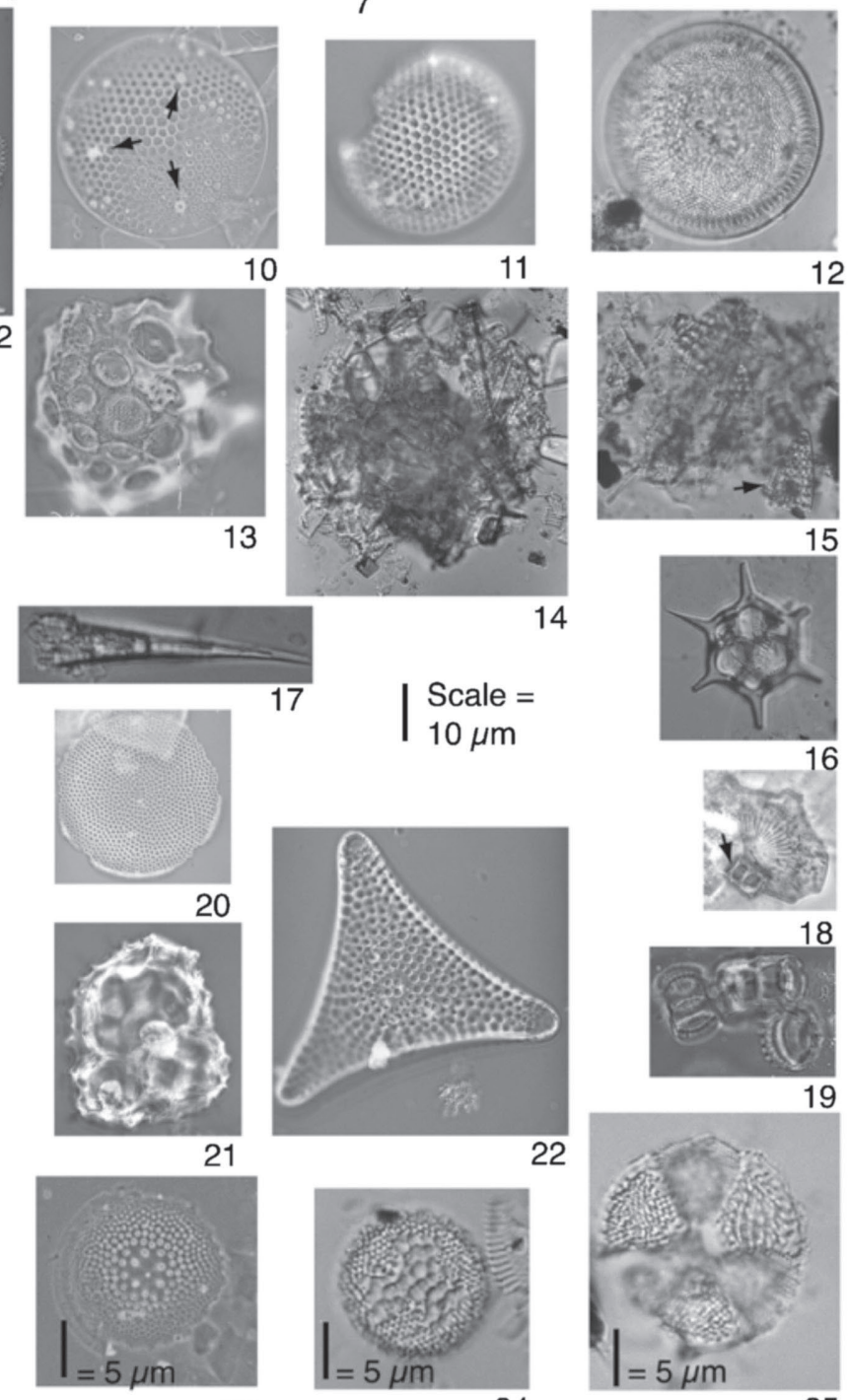

23
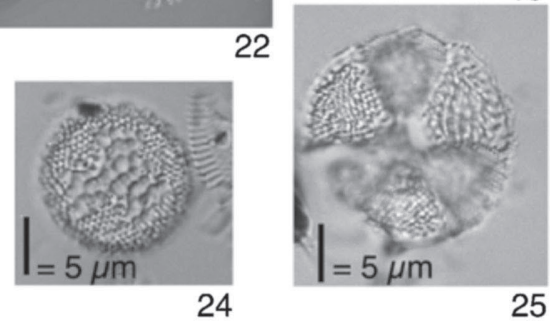

tion of the associated freshwater taxa in sample PCM 90-11 makes this unlikely. The presence of $P$. barboi constrains the original (source bed) age to $<12.7 \mathrm{Ma}$.

\section{Battye Glacier Formation}

The sparse diatom fragments in the Battye Glacier Formation at Dragons Teeth (samples PCM 90-8 and PCM 90-10B: Figure 3c; Table 1) are of the extant planktic marine Thalassiothrix sp., the age range of which has not been established. The Dragons Teeth facies are basin-marginal, and are in marked contrast to the Battye Glacier Formation outcrops $13 \mathrm{~km}$ to the northeast (Whitehead et al. 2003), where more distal diatom-bearing muds contain up to $12 \%$ opal (samples 
PCM 00-118 and PCM 00-116: Figure 3c Section 2). These are the most biogenic facies known so far in the Pagodroma Group and their abundant benthic diatoms point to a shallow (euphotic) bathymetry. Because the opal content is comparable to that of contemporary marine sediment in Prydz Bay today, biostratigraphic analysis has been undertaken using a similar approach to that used upon open-marine strata, where the absence of taxa helps constrain the age (Figure 8a). That the two samples (PCM 00-116, PCM 00-118) were deposited at some time between 11.7 and 9.0 Ma (Middle to Late Miocene) is indicated by the presence of $D$. vulgaris (first occurrence 11.7-10.7 Ma) and by the absence of $T$. torokina (first occurrence 9.0 Ma) (Whitehead et al. 2003). Furthermore, the absence of $D$. dimorpha, which occurs nearby as a reworked component in the younger Bardin Bluffs Formation, suggests constraint of the age to less than ca $10.7 \mathrm{Ma}$ (Whitehead et al. 2003) (Figure 8).

\section{Bardin Bluffs Formation}

The abundant diatoms recovered from the older Member 1 mudstone (sample 20-12 of Bardin \& Belevich 1985) (Figure 3), include Actinocyclus actinochilus. This extant species (first occurrence $3.1 \mathrm{Ma}$ ), indicates that Member 1 was deposited $<3.1 \mathrm{Ma}$ (Figure $8 \mathrm{~b}$ ), and the presence of T. antarctica could even infer an age $<0.67 \mathrm{Ma}$ for Member 1 (Bohaty et al. 1998). However, this latter datum has been established from lower latitude oceanic occurrences on the Kerguelen Plateau (Scherer 1991), and it may become diachronously older southwards onto the Antarctic continental shelf where Thalassiosira antarctica is currently widespread, and ranges from rare to $15 \%$ relative abundance (Armand 1998). Antarctic continental shelf records are disparate and further work is required to determine the oldest age for the first occurrence of Thalassiosira antarctica.

An attempt has been made to relocate the field position of sample 20-12; sample PCM 98-3Aa-026 is considered to be close to the sample horizon. Both samples contain a high abundance of benthic diatom taxa, although planktic diatoms are common in sample 20-12. Unfortunately the scarcity of biostratigraphically well-constrained planktic taxa precludes a more precise age determination of the Bardin Bluffs Formation from sample PCM 98-3Aa-026. The high abundance of Antarctic benthic marine diatoms in both of these samples emphasises that deposition occurred in a shallow euphotic environment.

In the younger Member 1 mudstone (sample PCM 90-7: Figures $3,8 \mathrm{~b}$ ) with its moderately high abundance of intact and fragmented diatoms, A. actinochilus again indicates deposition <3.1 Ma. The presence of the foraminifer Globigerina sp. cf. G. antarctica in PCM 90-7 further constrains the age to between 2.6 and $0.99 \mathrm{Ma}$ (McKelvey et al. 2001). Together with the presence of Actinocyclus karstenii Van Heurck (last occurrence 2.9-1.8 Ma), these fossils potentially constrain the age of PCM 90-7 to between 2.6 and 1.8 Ma. The diatom assemblage is biostratigraphically mixed, with the co-occurrence of A. actinochilus (first occurrence 3.1) and F. praeinterfrigidaria (last occurrence $3.6 \mathrm{Ma}$ ). We consider F. praeinterfrigidaria to have been glacially reworked from Lambert Graben marine strata of between 5.8 and $3.6 \mathrm{Ma}$ in age, and incorporated into this younger Member 1 mudstone. (A) MT JOHNSTON
FORMATION

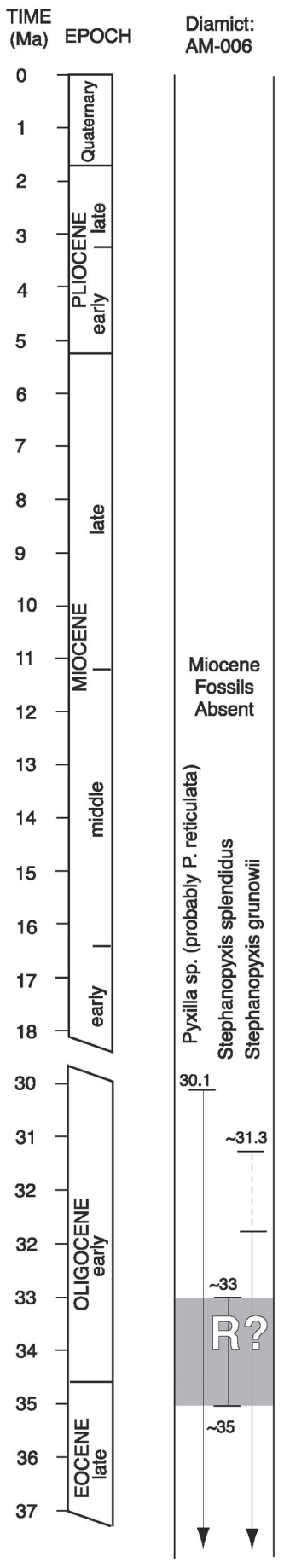

(B) FISHER BENCH FORMATION

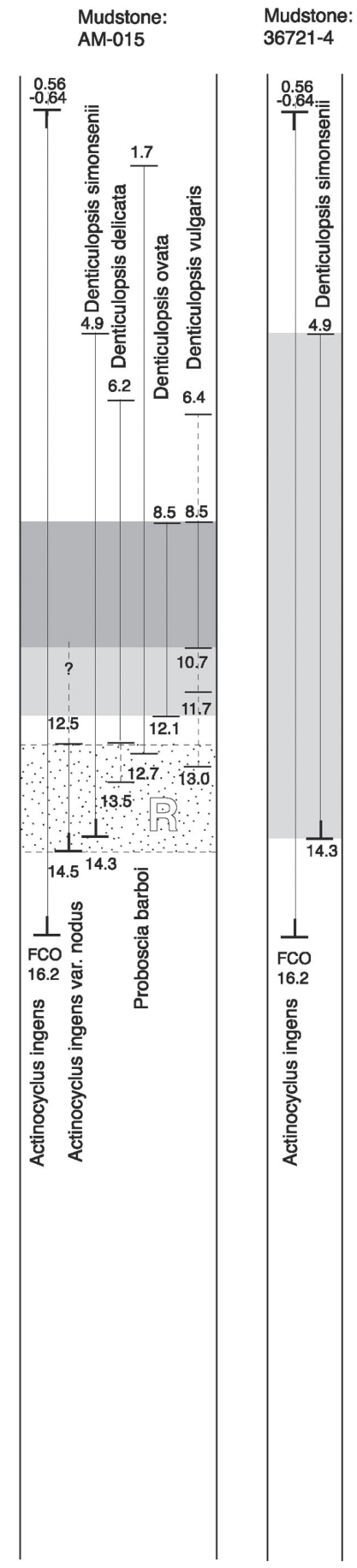

Figure 7. Age ranges inferred from diatom biostratigraphy of the Mt Johnston Formation (AM-006) and Fisher Bench Formation (AM015, 36721-4). Dark-grey hachure indicates the most constrained age interpretation. Light-grey hachure indicates broader age ranges from other reliable data. Stippled hachure indicates the time range of source areas from where non-in situ diatoms have been derived by glacial reworking. The symbol " $R$ ?" indicates uncertainty as to whether the sparse flora is in situ or else of a reworked origin. The Miocene fossils in the Fisher Bench Formation are absent from the Mt Johnston Formation. 


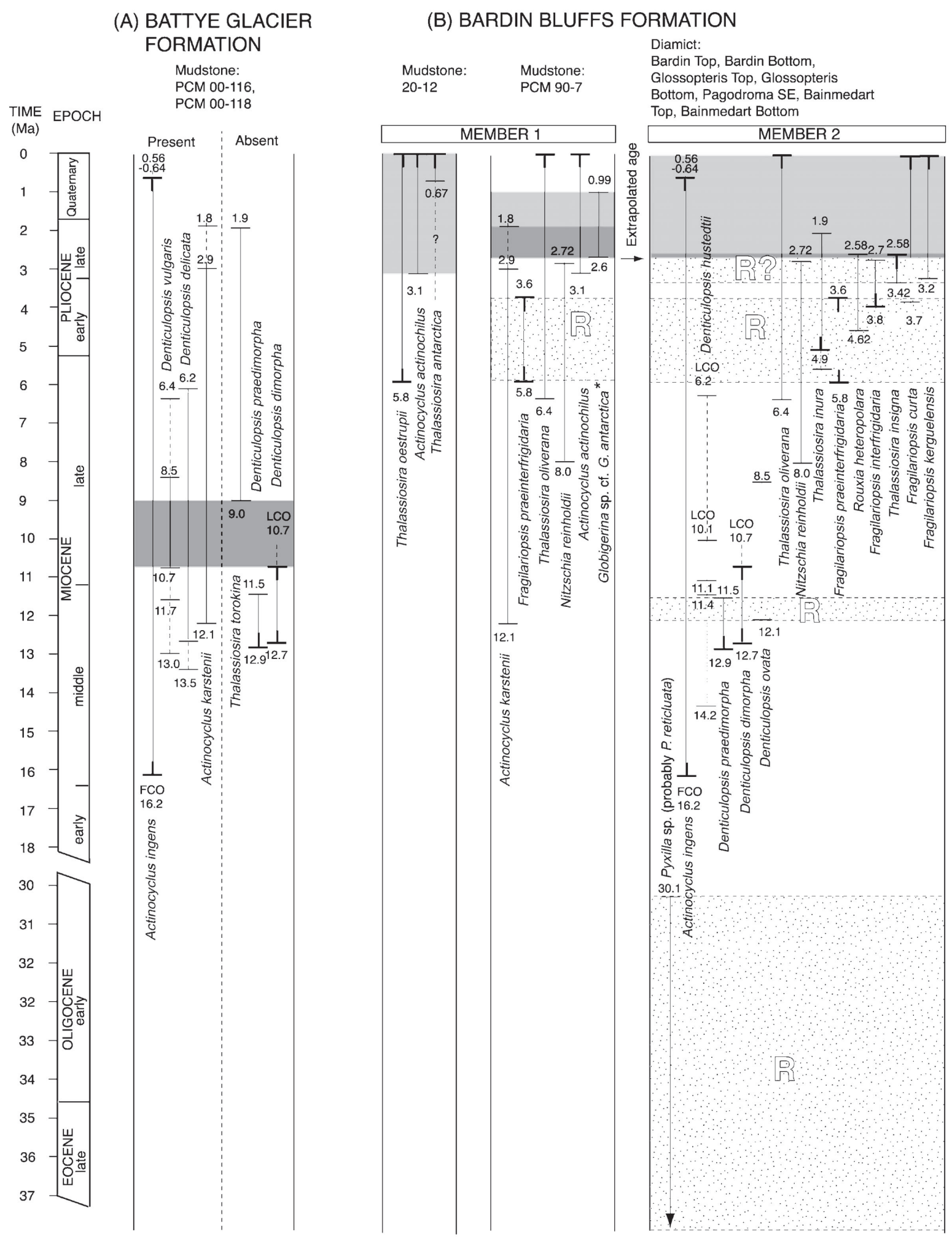

Figure 8. Age ranges inferred from diatom biostratigraphy (samples indicated) of the Battye Glacier and Bardin Bluffs Formations. (a) Battye Glacier Formation biostratigraphic interpretation is depicted by dark-grey hachure that constrains the age based upon "present" and "absent" diatoms. The diatom abundance in the Battye Glacier Formation is comparable to that of open-marine sediments, where the absence of diatom taxa is commonly used to help constrain age. (b) Bardin Bluffs Formation biostratigraphic interpretations for Member 1 and Member 2 are illustrated separately. Dark-grey hachure indicates the most constrained age interpretation from the diatoms present. Light-grey hachure indicates broader age ranges from other reliable diatom data. Stippled areas and the symbol " $R$ " indicates the time range of source areas from which non-in situ diatoms have been derived by glacial reworking. The symbol "R?" in Member 2 indicates uncertainty of reworking because T. insigna has a very small range overlap of 0.02 million years with the Member 1 basal datum extrapolation (i.e. G. sp. cf. G. antarctica* foraminiferal datum in McKelvey et al. 2001). 
Table 2. Biostratigraphic data used in the present study.

\begin{tabular}{|c|c|c|c|c|}
\hline Datum & Taxon & $\begin{array}{l}\text { Revised age } \\
(\mathrm{Ma})^{\mathrm{b}}\end{array}$ & Source(s) & Other reported ages/notes \\
\hline \multicolumn{5}{|c|}{ Zonal markers } \\
\hline $\mathrm{LCO}$ & Actinocyclus ingens & $0.56-0.64$ & ZG & $\begin{array}{l}\text { B: } 0.40-0.55^{\mathrm{a}} / \mathrm{RB}: 0.3-2.0^{\mathrm{b}} / \mathrm{WI}: 0.65^{\mathrm{b}} / \\
\quad \text { ZG: } 0.38^{\mathrm{b}} \text { (LO) / C, G, HM, GB: } 0.66^{\mathrm{b}} \text { (LCO) }\end{array}$ \\
\hline LO & Thalassiosira insigna & 2.6 & C / GB & $\begin{array}{l}\text { G: } 2.1-2.5^{\mathrm{a}} / \text { F: } 1.9-2.2^{\mathrm{a}} / \text { B: } 2.4-2.45^{\mathrm{a}} / \\
\quad \text { RB: } 1.8-4.6^{\mathrm{b}} / \text { WI: } 2.54-2.6^{\mathrm{b}} / \text { ZG: } 2.55-2.66^{\mathrm{b}}\end{array}$ \\
\hline $\mathrm{FO}$ & Fragilariopsis interfrigidaria & 3.8 & $\mathrm{G} / \mathrm{B} / \mathrm{HM} / \mathrm{ZG}$ & C: $4.0^{\mathrm{a}} / \mathrm{RB}: 3.3-4.0^{\mathrm{b}} / \mathrm{WI}: 2.67-3.9^{\mathrm{b}}$ \\
\hline FO & Thalassiosira oestrupii & 6.2 & WI & RB: $4.3-5.1^{\mathrm{b}} / \mathrm{B}: 5.6-5.8^{\mathrm{b}}$ \\
\hline $\mathrm{FO}$ & Thalassiosira torokina & 9.0 & $\mathrm{HM}$ & C: $\sim 6.0^{\mathrm{a}} / \mathrm{G}: \sim 7.7^{\mathrm{a}} / \mathrm{B}: 7.7^{\mathrm{a}} / \mathrm{RB}: 8.5-10.0^{\mathrm{b}}$ \\
\hline $\mathrm{LCO}$ & Denticulopsis dimorpha & 10.7 & $\mathrm{HM}$ & G: $9.8-9.9^{a} /$ B: $9.9^{a} /$ RB: $10.2-12.9^{b} /$ YA: $9.2^{b}(L O)$ \\
\hline $\mathrm{FO}$ & Denticulopsis dimorpha & 12.7 & CG & G: $12.4^{\mathrm{a}} / \mathrm{RB}: 11.1-13.0^{\mathrm{b}} / \mathrm{B}, \mathrm{HM}: 12.2^{\mathrm{b}}$ \\
\hline FO & Denticulopsis praedimorpha & 12.9 & CG & $\begin{array}{l}\text { B: } 12.0-12.5^{\mathrm{a}} / \mathrm{HM}: 12.8^{\mathrm{a}} / \mathrm{RB}: 12.5-13.4^{\mathrm{b}} / \\
\text { /G: } 12.82^{\mathrm{b}}\end{array}$ \\
\hline FO & Denticulopsis simonsenii & $14.18-14.3$ & CG & $\begin{array}{l}\text { B: } 14.0^{\mathrm{a}} \text { (FCO) / RB: } 14.0-14.6^{\mathrm{b}} / \text { YA: } 14.5-14.6^{\mathrm{b}} \\
\text { (Northern Hemisphere) }\end{array}$ \\
\hline \multicolumn{5}{|c|}{ Secondary data } \\
\hline $\mathrm{FO}$ & Thalassiosira antarctica & $<0.67$ & S / B1+ & - \\
\hline $\mathrm{LO}$ & Globigerina sp. cf. G. antarctica ${ }^{\mathrm{c}}$ & 0.99 & $\mathrm{M}$ & - \\
\hline LO & Proboscia barboi & 1.7 & $\mathrm{~F} / \mathrm{C} / \mathrm{B}$ & $\begin{array}{l}\text { HM: } 3.1^{\mathrm{a}} \text { (local extinction) / RB: } 1.6-4.2^{\mathrm{b}} / \\
\text { ZG: } 1.82-1.85^{\mathrm{b}}\end{array}$ \\
\hline $\mathrm{LO}$ & Thalassiosira inura & 1.9 & $\mathrm{HM} / \mathrm{W}$ & G: $1.7^{\mathrm{a}} / \mathrm{RB}: 2.0-3.1^{\mathrm{b}} / \mathrm{WI}: 1.97^{\mathrm{b}}$ \\
\hline $\mathrm{FO}$ & Globigerina sp. cf. G. antarctica \# & $\neq 2.6$ & $\mathrm{M}$ & - \\
\hline $\mathrm{LO}$ & Rouxia heteropolara & 2.58 & G & $\mathrm{RB}: 2.2^{\mathrm{b}}$ \\
\hline LO & Fragilariopsis interfrigidaria & 2.7 & $\mathrm{HM}$ & $\begin{array}{l}\text { C: } 2.8^{\mathrm{a}} / \mathrm{G}: 2.1-2.5^{\mathrm{a}} / \mathrm{B}: 1.9-2.0^{\mathrm{a}} / \mathrm{RB}: 1.8-3.3^{\mathrm{b}} / \\
\text { ZG: } 2.58-2.96^{\mathrm{b}}\end{array}$ \\
\hline $\mathrm{FO}$ & Actinocyclus actinochilus & 3.1 & HM & RB: $2.2-3.2^{\mathrm{b}}$ \\
\hline $\mathrm{FO}$ & Fragilariopsis kerguelensis & 3.2 & $\mathrm{HM}$ & C: $2.6^{\mathrm{a}} /$ B: $1.9-2.0^{\mathrm{a}} / \mathrm{RB}: 1.2-3.2^{\mathrm{b}} /$ ZG: $2.04-2.34^{\mathrm{b}}$ \\
\hline LO & Denticulopsis delicata & 6.2 & BW & - \\
\hline FO & Thalassiosira oliverana & 6.4 & HM & - \\
\hline $\mathrm{FO}$ & Nitzschia reinholdii & 8.0 & $\mathrm{~B}$ & HM: $6.4^{\mathrm{b}}$ \\
\hline LO & Denticulopsis ovata & $\sim 9.0$ & $\mathrm{HM}$ & CG: $4.93^{\mathrm{b}}$ \\
\hline $\mathrm{LCO}$ & Denticulopsis hustedtii & 10.1 & YA & $\begin{array}{l}\text { HM: } 6.2^{\mathrm{b}} \text { (when grouped with } D \text {. simonsenii } \\
\text { and D. vulgaris) }\end{array}$ \\
\hline $\mathrm{FO}$ & Denticulopsis vulgaris & $10.7-11.7$ & $\mathrm{~B} 2+$ & YA: $13.0^{\mathrm{b}}$ (Northwest Pacific datum) \\
\hline $\mathrm{FO}$ & Denticulopsis hustedtii & $11.1-11.4$ & YA & $\begin{array}{l}\text { HM: } 14.2^{\mathrm{b}} \text { (when grouped with } D \text { simonsenii } \\
\text { and D. vulgaris) }\end{array}$ \\
\hline $\mathrm{LO}$ & Denticulopsis praedimorpha & 11.5 & $\mathrm{~B} / \mathrm{HM}$ & C: $10.5^{a} /$ G: $10.4^{a} /$ RB: $10.9-12.1^{b}$ \\
\hline $\mathrm{FO}$ & Denticulopsis ovata & 12.11 & CG & HM: $11.7^{\mathrm{b}}$ \\
\hline FO & Actinocyclus karstenii & 12.1 & HM & - \\
\hline LO & Actinocyclus ingens var. nodus & 12.5 & $\mathrm{G} / \mathrm{HM}$ & B: $12.0-12.5^{a} / R B: 9.3-14.6^{b}$ \\
\hline $\mathrm{FO}$ & Denticulopsis delicata & $12.5-13.5$ & BW & - \\
\hline FO & Proboscia barboi & 12.7 & $\mathrm{HM}$ & RB: $11.4-11.7^{b}$ \\
\hline LO & Stephanopyxis splendidus & $\sim 33$ & HB & - \\
\hline LO & Pyxilla reticulata & 30.1 & S & - \\
\hline LO & Stephanopyxis grunowii & $\sim 31.3-32$ & GC & - \\
\hline $\mathrm{FO}$ & Stephanopyxis splendidus & $\sim 35$ & HB & GC: $\sim 34^{b}$ \\
\hline
\end{tabular}

C, Ciesielski (1983); G, Gersonde \& Burckle (1990); F, Fenner (1991); B, Baldauf \& Barron (1991); HM, Harwood \& Maruyama (1992); WH, Winter \& Harwood (1997); WI, Winter \& Iwai (2002); YA, Yanagisawa \& Akiba (1998); RB, Ramsay \& Baldauf (1999); S, Scherer 1991; B1+, Bohaty et al. (1998); B2+, Bohaty et al. (2003); CG, Censarek \& Gersonde (2002); ZG, Zielinski \& Gersonde (2002) data south of Subantarctic Front; M, McKelvey et al. 2001; HB, Harwood \& Bohaty (2001); GC, Gombos \& Ciesielski (1983); BW, S. M. Bohaty \& J. M. Whitehead (unpubl. data); S+, Scherer et al. (2000) a. Berggren et al. (1985); b. Berggren et al. (1995) / Cande \& Kent (1995); c. Foraminiferal data (McKelvey et al. 2001)

FO, First Occurrence; FCO, First Common Occurrence; LO, Last Occurrence; LCO, Last Common Occurrence.

Data in italics are considered by the original authors to be of low reliability. 
Figure 9. Diatomaceous clasts or aggregates are of similar particle size to aeoliantransported diatoms sampled from Southern Ocean islands (Chalmers et al. 1996) and continental loess. All three groups exceed the particle size of aeolian dust in Antarctic ice cores. However, numerous diatomaceous clasts recovered from PCM 90-2 are considerably larger than any of the four categories illustrated (figure modified from Harwood \& Webb 1998).

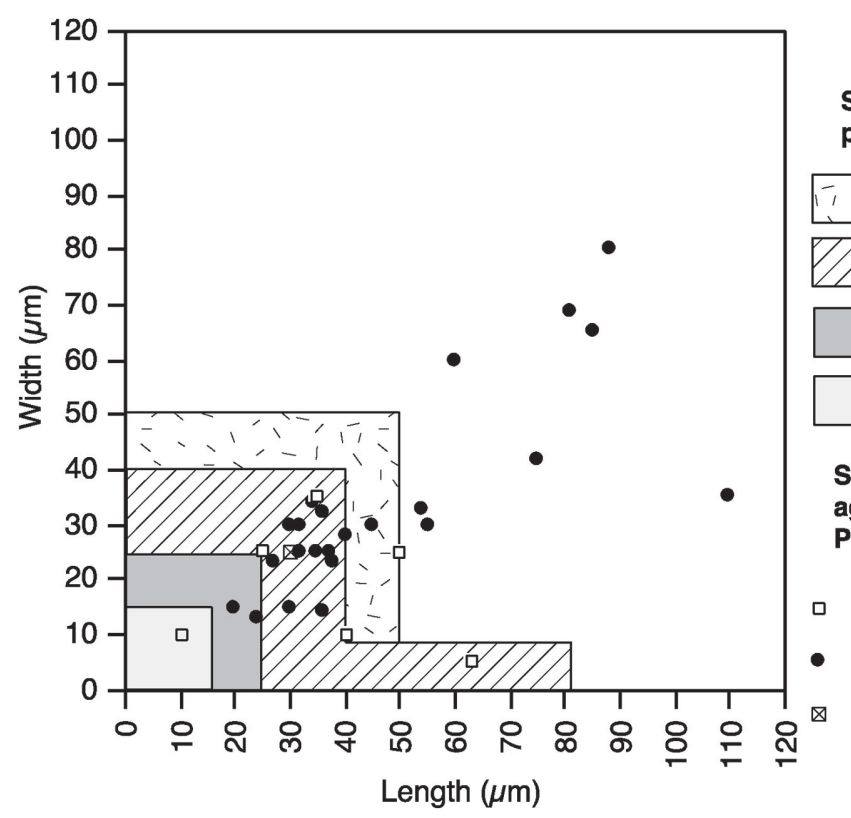

KEY

Size of windblown particles

Continental loess

Eolian diatoms, from Southern Ocean islands Dust in Antarctic ice cores Eolian component in the deep sea

Size of diatomaceous aggregates in the Pagodroma Group

Bardin Bluffs Fm Diamicts PCM 90-2 PCM 90-11

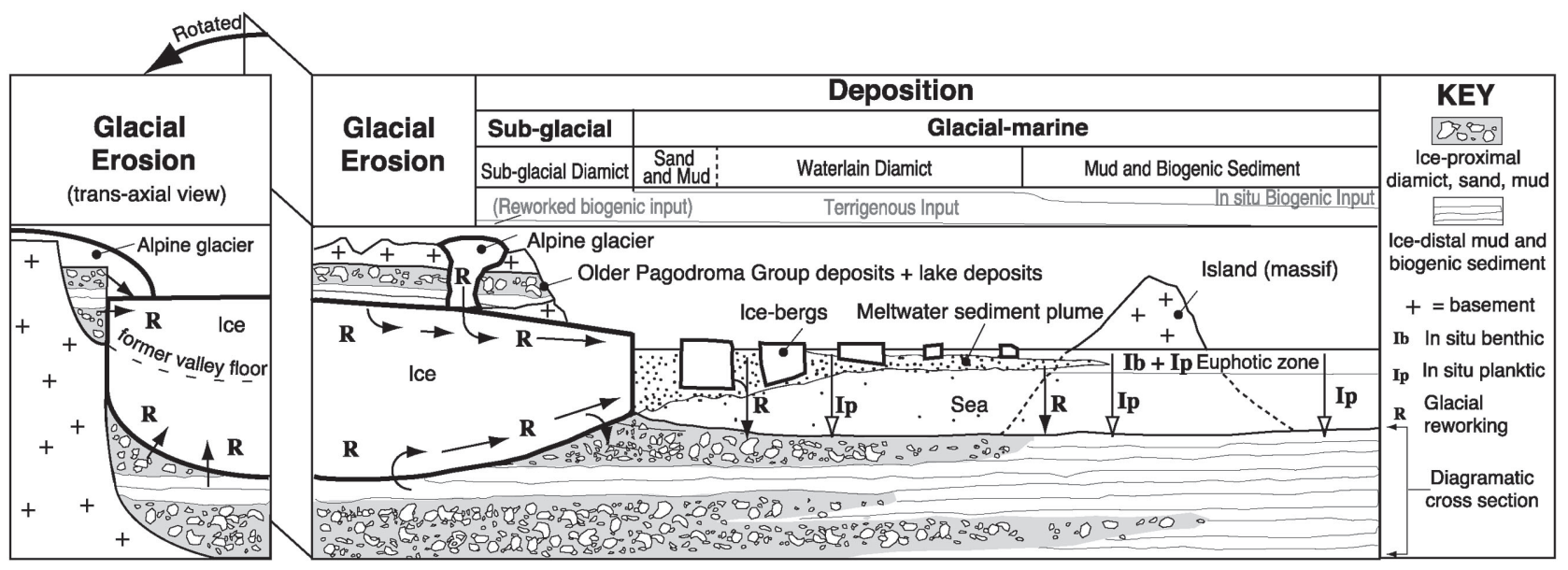

Figure 10. Pagodroma Group erosional and depositional scenario, as proposed by Hambrey and McKelvey (2000a, b), redrawn to incorporate microfossil emplacement by glacial reworking and in situ marine deposition. Possible glacial reworking pathways, depicted by "R," include marine sediment eroded from beneath the Lambert Glacier, and similarly eroded by alpine and tributary glaciers. Reworked microfossils are deposited into subglacial diamict, and beyond the ice terminus reworked microfossils are deposited from meltwater plumes and from icebergs into sand, mud and water-lain diamict. Some in situ microfossil assemblages also contain older reworked taxa. In situ planktic and benthic marine microfossil deposition occurs in the marine environment although relatively biogenic-rich sediments accumulate only in icedistal settings. The island illustrated may represent the Amery Oasis, and other massifs of the Prince Charles Mountains, during reduced glacial conditions.

Ecological differences between the diatom assemblages in samples 20-12, PCM 98-3Aa-026 and PCM 90-7 suggest a considerable sea-level rise during deposition of Member 1. Samples 20-12 and PCM 98-3Aa-026 contain many shallow marine benthic diatoms, yet sample PCM 90-7 consists almost exclusively of planktic taxa (Table 1). The low abundance of benthic diatoms in the latter sample suggests deposition beneath euphotic depth. The foraminiferal assemblage in PCM 90-7 similarly reflects a deep-water environment (McKelvey et al. 2001). This relative transgression is further supported by the presence of a (subaerial) palaeosol on the unconformity surface beneath Member 1 at Bardin Bluffs (Whitehead \& McKelvey 2001).

Diatoms from Member 1 mudstone at the "Hiatella location" sample (PCM 90-2: Figure 3d; Table 1) consist largely of extant planktic species, with the exception of extinct planktic taxa A. ingens (last occurrence $0.64-0.56 \mathrm{Ma}$ ) and $R$. isopolica. The presence of Thalassiosira oliverana ( $\mathrm{O}^{\prime}$ Meara) Sournia (first occurrence 6.4 Ma) indicates deposition after 6.4 Ma. However, the absence of $A$. actinochilus prevents a more precise comparison with the two mudstones at Bardin Bluffs. Many diatoms in sample PCM 90-2 occur in diatomaceous sediment clasts that are too large to be aeolian contaminants (Figure 9), suggesting that they are reworked glacially from an older marine sediment source.

In Member 2 diamicts of the Bardin Bluffs Formation the presence of F. kerguelensis (first occurrence 3.2 Ma) in samples from "Bardin Bottom," "Glossopteris Top," "Glossopteris Bottom," "Pagodroma SE," and "Bainmedart Bottom" (Figure $3 b$ ), indicates deposition <3.2 Ma. Their stratigraphic 
Table 3. Criteria for assessing diatom emplacement within the Pagodroma Group.

\begin{tabular}{|c|c|c|c|c|c|c|c|c|c|c|c|c|c|c|}
\hline \multirow{2}{*}{$\begin{array}{l}\text { Formations } \\
\text { Sample Location }\end{array}$} & \multicolumn{11}{|c|}{ Bardin Bluffs } & \multicolumn{3}{|c|}{ Battye Glacier } \\
\hline & 1 & 1 & 5 & 5 & 3 & 4 & 4 & 2 & 1 & 1 & 1 & 6 & 6 & 6 \\
\hline Sample Name & Bardin & Bardin & Gloss & Gloss & Pago & Bain & Bain & PCM & PCM & PCM & PCM 98 & PCM & PCM & PCM \\
\hline Sample Position/Number & & bottom & top & bottom & SE & top & bottom & $90-2$ & $90-3$ & $90-7$ & $3 \mathrm{Aa}-26$ & $90-8$ & $90-10 \mathrm{~A}$ & $90-10$ \\
\hline Lithology & $\mathrm{Dm}$ & $\mathrm{Dm}$ & $\mathrm{Dm}$ & $\mathrm{Dm}$ & $\mathrm{Dm}$ & $\mathrm{Dm}$ & $\mathrm{Dm}$ & Silt. & $\mathrm{Dm}$ & Silt. & Silt. & $\mathrm{Dm}$ & $\mathrm{Dm}$ & $\mathrm{Dm}$ \\
\hline Diatom abundance & 3 & 4 & 4 & 3 & 4 & 3 & 3 & 4 & 0 & 5 & 6 & 1 & 0 & 1 \\
\hline $\begin{array}{l}\text { Raw or concentrated slides } \\
\text { Biogenic silica (\% opal) }\end{array}$ & conc. & conc. & conc. & conc. & conc. & conc. & conc. & $\begin{array}{l}\text { conc. } \\
2 \%\end{array}$ & conc. & $\begin{array}{l}\text { conc. } \\
1 \%\end{array}$ & $\begin{array}{l}\text { raw } \\
2 \%\end{array}$ & conc. & conc. & conc. \\
\hline \multicolumn{15}{|l|}{ Airborne Contamination } \\
\hline \multicolumn{15}{|l|}{ From aeolian deposition } \\
\hline $\begin{array}{l}\text { Predominantly } \\
\quad \text { fresh-water diatoms }\end{array}$ & $\mathrm{r}$ & $\mathrm{r}$ & $\mathrm{r}$ & $\mathrm{r}$ & $\mathrm{r}$ & $\mathrm{r}$ & $\mathrm{r}$ & - & - & $\mathrm{r} ?$ & - & - & - & - \\
\hline $\begin{array}{l}\text { Predominantly } \\
\text { benthic diatoms }\end{array}$ & $\mathrm{r}$ & - & $\mathrm{r}$ & $\mathrm{r}$ & $\mathrm{r}$ & - & $\mathrm{r} ?$ & - & - & $\mathrm{r}$ & $x$ & - & - & - \\
\hline \multicolumn{15}{|l|}{ From meteorite impact fallout } \\
\hline $\begin{array}{l}\text { Predominantly planktic } \\
\text { with mixed age range } \\
\text { (ca } 50-2.15 \mathrm{Ma})\end{array}$ & - & - & - & - & $\underline{X} ?$ & - & - & - & - & - & - & - & - & - \\
\hline \multicolumn{15}{|l|}{ Supporting evidence } \\
\hline Diatomaceous aggregates & - & $x$ & $x$ & $X$ & $x$ & - & $x$ & $x$ & - & - & - & - & - & - \\
\hline \multicolumn{15}{|l|}{ Glacial Reworking } \\
\hline \multicolumn{15}{|l|}{ From marine strata } \\
\hline $\begin{array}{l}\text { Marine Southern Ocean } \\
\text { and Antarctic species }\end{array}$ & $\underline{x}$ & $\underline{x}$ & $\underline{x}$ & $\underline{x}$ & $\underline{x}$ & $x$ & $\underline{x}$ & $\underline{x}$ & - & $\underline{x}$ & $x$ & $x$ & - & $x$ \\
\hline \multicolumn{15}{|l|}{ Supporting evidence } \\
\hline Diatomaceous aggregates & - & $\underline{x}$ & $\underline{x}$ & $\underline{x}$ & $\underline{x}$ & - & $\underline{x}$ & $\underline{x}$ & - & - & - & - & - & - \\
\hline $\begin{array}{l}\text { Diatoms biostratigraphi- } \\
\text { cally mixed (different } \\
\text { ages together) }\end{array}$ & $\underline{x}$ & - & $\underline{x}$ & $\underline{x}$ & $\underline{x}$ & - & $\underline{x}$ & - & - & $\underline{x}$ & - & - & - & - \\
\hline \multicolumn{15}{|l|}{ From lake strata } \\
\hline Freshwater species & $\mathrm{r}$ & $\mathrm{r}$ & $\mathrm{r}$ & $\mathrm{r}$ & $\mathrm{r}$ & $\mathrm{r}$ & $\mathrm{r}$ & - & - & $\mathrm{r} ?$ & - & - & - & - \\
\hline \multicolumn{15}{|l|}{ Supporting evidence } \\
\hline $\begin{array}{l}\text { Freshwater diatomaceous } \\
\text { aggregate }\end{array}$ & - & - & - & - & - & - & $x$ & - & - & - & - & - & - & - \\
\hline \multicolumn{15}{|l|}{ In situ Marine } \\
\hline $\begin{array}{l}\text { Marine Southern Ocean } \\
\text { and Antarctic species }\end{array}$ & $x$ & $x$ & $x$ & $x$ & $x$ & $x$ & $x$ & $\underline{x}$ & - & $\underline{x}$ & $\underline{x}$ & $x$ & - & $x$ \\
\hline \multicolumn{15}{|l|}{ Supporting evidence } \\
\hline In situ molluscs & - & - & - & - & - & - & - & $x$ & - & $x$ & - & - & - & - \\
\hline $\begin{array}{l}\text { Diatoms relatively } \\
\text { abundant }^{\mathrm{a}}\end{array}$ & - & - & - & - & - & - & - & - & - & - & $x$ & - & - & - \\
\hline $\begin{array}{l}\text { Diatoms biostratigraphi- } \\
\text { cally unmixed (coherent } \\
\text { stratigraphic range) }\end{array}$ & - & $x$ & - & - & - & $x$ & - & $x$ & - & - & $x$ & $x$ & - & $x$ \\
\hline
\end{tabular}

position above Member 1 at Bardin Bluffs further constrains the age to $<2.6 \mathrm{Ma}$.

Several of the Bardin Bluffs Formation diamicts contain diatoms with considerably disparate age ranges (Figure 8). These data suggest glacial reworking. Thus the Eocene-Oligocene $P$. reticulata may have originated from a source similar to that of the Pyxilla sp. found in the Mt Johnston Formation. Reworked Miocene marine diatoms in the diamicts include $D$. dimorpha and $D$. praedimorpha, which lived be- tween 12.9 and $c a 10.7 \mathrm{Ma}$. These species have not been recovered from the Miocene Fisher Bench and Battye Glacier Formations, and so their presence in the younger Bardin Bluffs Formation suggests an earlier phase of Miocene marine deposition in the Lambert Graben. As in the Member 1 mudstones (see above) F. praeinterfrigidaria in the diamicts records marine deposition in the Lambert Graben at some time between 5.8 and 3.6 Ma. Similarly the presence of T. insigna in Member 2 may record Pliocene deposition between 3.4 


Fisher Bench

6
AM
028
Dm
0
conc.
-

\begin{tabular}{llllll}
\hline 9 & 9 & 9 & 9 & 8 & 7 \\
PCM & PCM & PCM & AM & AM & AM \\
$90-6$ & $90-11$ & $90-13$ & 015 & 017 & 026 \\
Silt. & Dm & Dm & Silt. & Dm & Dm \\
2 & 4 & 0 & 6 & 4 & 1 \\
conc. & conc. & conc. & raw & conc. & conc. \\
$1 \%$ & $1 \%$ & - & $\sim 2 \%$ & - & $1 \%$
\end{tabular}

\section{Mt Johnston}

\begin{tabular}{|c|c|c|c|c|c|c|c|c|c|}
\hline & \\
\hline & & & & & & Bardin & Battye & & Fisher \\
\hline 10 & 10 & 10 & 10 & 10 & 10 & 1 & 11 & 11 & 9 \\
\hline PCM & PCM & $\mathrm{AM}$ & AM & $\mathrm{AM}$ & $\mathrm{AM}$ & & PCM & PCM & \\
\hline $90-4$ & $90-5$ & 002 & 003 & 006 & 014 & $20-12$ & $00-116$ & $00-118$ & $36721-4$ \\
\hline $\mathrm{Dm}$ & $\mathrm{Dm}$ & $\mathrm{Dm}$ & $\mathrm{Dm}$ & $\mathrm{Dm}$ & $\mathrm{Dm}$ & Silt. & Silt. & Silt. & Silt. \\
\hline 2 & 2 & 0 & 1 & 2 & 2 & 6 & 6 & 6 & 5 \\
\hline conc. & conc. & conc. & conc. & conc. & conc. & raw & raw & raw & conc. \\
\hline- & - & - & - & - & - & - & $7 \%$ & $12 \%$ & - \\
\hline
\end{tabular}

\begin{tabular}{|c|c|c|c|c|c|c|c|c|c|c|c|c|c|c|c|}
\hline$X$ & $x$ & $x$ & - & $X$ & $x$ & $X$ & $X$ & $x$ & - & $X$ & $X$ & $x$ & $X$ & $X$ & $X$ \\
\hline - & - & - & - & - & - & - & - & - & - & - & - & - & - & - & - \\
\hline - & - & - & - & $?$ & - & - & - & - & - & - & - & - & - & - & - \\
\hline- & - & $\mathrm{r}$ & - & - & - & - & - & - & - & - & - & - & - & - & - \\
\hline - & - & - & - & - & - & - & - & - & - & - & - & - & - & - & - \\
\hline$x$ & $x$ & $X$ & - & $X$ & $x$ & $x$ & $x$ & $x$ & - & $x$ & $x$ & $x$ & $X$ & $x$ & $X$ \\
\hline - & - & - & - & - & - & - & - & - & - & - & - & - & - & $X$ & $X$ \\
\hline - & - & - & - & $x$ & - & - & - & - & - & - & - & - & $x$ & $x$ & $X$ \\
\hline$X$ & $x$ & $x$ & - & $?$ & $x$ & $X$ & $x$ & $x$ & - & $x$ & $X$ & $x$ & $X$ & $x$ & $x$ \\
\hline
\end{tabular}

and 2.6 Ma. Alternatively, because T. insigna has a 0.02 million year overlap with the youngest age assigned to Member 1 of the Bardin Bluffs Formation (from G. sp. cf. G. antarctica, first occurrence 2.6 Ma), its presence could infer a restricted in situ formation age between 2.6 and $2.58 \mathrm{Ma}$. However, this interpretation is probably beyond the resolution of the biostratigraphic dating method.

The freshwater planktic diatoms Stephanodiscus sp., Cyclotella sp. and T. fenestrata in the Bardin Bluffs Formation Member 2 diamicts ("Bardin Top," "Glossopteris Top," "Pagodroma SE," and "Bainmedart Bottom" samples: Figure 3), indicate a post-Late Miocene age for former lake sediments (Bradbury \& Krebs 1995) in the Lambert Graben catchment.

\section{Conclusions}

Diatom-bearing marine deposits of the Pagodroma Group formed during periods of reduced glacial extent when marine waters penetrated southwards into the Lambert Graben. The two primary modes of diatom emplacement into the Pagodroma Group are (i) in situ marine deposition and (ii) glacial reworking of older microfossil-bearing marine and lacustrine strata, as illustrated in Figure 10. In situ marine deposition is demonstrated by the relative abundance of diatoms in mudstone sequences bounded by diatom-poor diamicts. Because these mudstones contain few dropstones, we consider them to be the facies most distal from the fjordal ice fronts (Hambrey \& McKelvey 2000b) of 
the ancestral Lambert Glacier - Amery Ice Shelf drainage system.

The in situ deposition of diatoms is further demonstrated (in the Battye Glacier Formation) by the close association of microfossil-bearing mudstones with strata containing fossil molluscs preserved in growth positions. The emplacement of diatoms by glacial reworking is indicated by biostratigraphic age mixing (Figures 7,8 ) and by the presence of some diatomaceous clasts that are too large to be aeolian contaminants (Figure 9). The relative insignificance of any aeolian contribution is highlighted by the fact that Pagodroma Group diatom assemblages (with few exceptions) are predominantly of planktic marine taxa. Freshwater taxa are rare. This contrasts with the aeolian diatom contribution in Antarctic ice cores (of South American provenance), which consists predominantly of marine benthic and non-marine species. Airborne diatom emplacement from the Eltanin asteroid impact ejecta is also unlikely. The Eltanin impact reworked Southern Ocean sediment spanning 50-2.15 Ma, and the resulting marine ejecta would be a biostratigraphically mixed assemblage. Those Pagodroma Group assemblages that are biostratigraphically mixed span only a small portion of that 50$2.15 \mathrm{Ma}$ range.

Biostratigraphic interpretation of the Pagodroma Group suggests that at least six episodes of marine deposition occurred in the Lambert Graben $>250 \mathrm{~km}$ south of the modern Amery Ice Shelf edge (Figure 11). Planktic marine diatoms in diamict of the Mt Johnston formation indicate an Early Oligocene age of between ca 35 and $33 \mathrm{Ma}$, but it is unclear if these fossils are in situ or glacially reworked. However, the lack of Miocene fossils and the formation's clearly older (field) relationship to the nearby Fisher Bench Formation (McKelvey et al. 2001) indicates that this formation predates the Middle to Late Miocene (>12.1 Ma). Direct evidence of two other episodes comes from the interpretation that in situ diatoms occur in mudstones of (i) the Battye Glacier and Fisher Bench Formations and (ii) the Bardin Bluffs Formation. The Battye Glacier and Fisher Bench Formations are broadly correlative with constrained ages between 10.7 and 9.0 Ma, and 10.7 (or 12.1) and 8.5 Ma, respectively. Foraminifers and diatoms in mudstone from the Bardin Bluffs

Figure 11. Summary of the diatom biostratigraphic interpretation of the Pagodroma Group. At least six marine intervals in the Lambert Graben are evident from in situ and glacially reworked diatoms from Fisher Massif and the Amery Oasis. Symbols and shading as in Figures 4, 5. Marine interval 1 is identified from diatoms (either in situ or glacially reworked) in the Mt Johnston Formation. The taxa fall within the age range of the oldest reworked fossils in the Bardin Bluffs Formation. Interval 2 is evident from reworked diatoms in the Fisher Bench Formation. Interval 3 is identified from reworked diatoms in the Bardin Bluffs Formation. Interval 4 is evident from in situ diatoms in the similarly aged Fisher Bench and Battye Glacier formations. (The age of the Battye Glacier Formation is also constrained by the conspicuous absence of some diatoms.) Interval 5 is identified from reworked diatoms in the Bardin Bluffs Formation. Interval 6 is evident from in situ microfossils in the Bardin Bluffs Formation. In theory it is possible to yet further constrain the age of the Bardin Bluffs Formation to 2.6-2.58 Ma. Alternatively, a seventh interval of marine sedimentation, slightly older than the Bardin Bluffs Formation, can be contemplated.

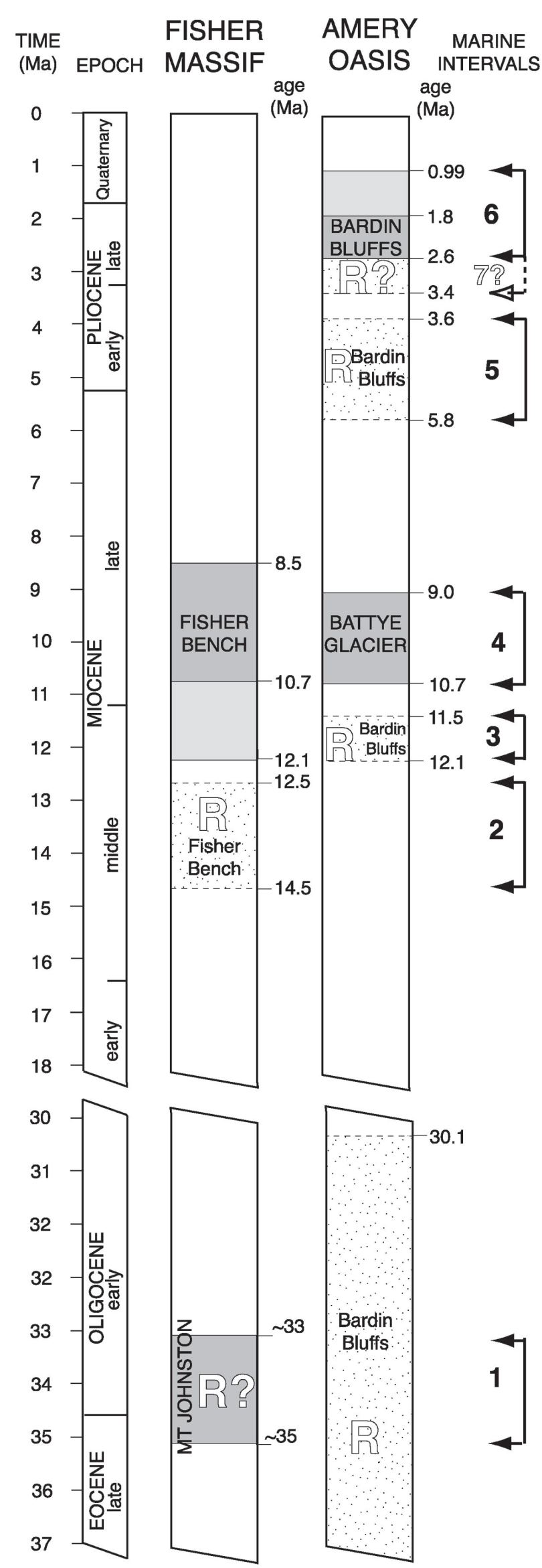


Formation indicate an age between 2.6 and $0.99 \mathrm{Ma}$, which may (possibly) be constrained further to 2.6-1.8 Ma. Indirect evidence for four other episodes of marine deposition comes from the identification of glacially reworked diatoms in the Bardin Bluffs Formation, and the Fisher Bench Formation, derived from 14.5 to $12.5 \mathrm{Ma}, 12.1-11.5,5.8-3.6 \mathrm{Ma}$ and, perhaps, 3.42-2.58 Ma strata (Figure 11).

It is to be emphasised, given the known rates of modern east Greenland fjordal sedimentation (Dowdeswell et al. 1994), that the episodes of marine sedimentation recorded by the formations did not persist throughout the time intervals defined by their diatom biostratigraphies. The individual Pagodroma Group formations in all probability represent time intervals in the order of only hundreds or thousands of years. Although six depositional episodes are identified, the 40000 year and 100000 year cyclicity established for such events elsewhere in Antarctica (Naish et al. 2001) suggest that over the $35 \mathrm{Ma}$ timespan indicated by the diatom biostratigraphy, the occurrence of some hundreds of such events is to be contemplated.

\section{Acknowledgments}

Samples for the present study were collected during several Australian National Antarctic Research Expeditions (ANARE) supported by Antarctic Science Advisory Committee (ASAC) Grants 9553871 499, 2086, and 1065. The Australian Antarctic Division is thanked for its splendid logistical support, especially that given through the personnel of Davis and Mawson stations. The air transport provided by Helicopter Resources (Australia) and Helicopters Australia was exceptional. Additional support was provided through NSF award OPP-0091676, OPP-0089899, and ARC award DP 0345161. Thanks to M. A. Harper for assistance with non-marine diatom taxonomy, and to Patrick Quilty, Fiona Taylor, James Etienne, Peter Barrett, Phillip O'Brien, and Jacob John for reviewing an early draft of this paper.

\section{References}

Adamson D., and Darragh A. 1991. Field evidence on Cainozoic history and landforms in the Northern Prince Charles Mountains, East Antarctica. In: Gillieson D. J., and Fitzsimons S. J. eds. Quaternary Research in Australian Antarctica: Future Directions, pp. 5-14. Department of Geography and Oceanography, Australian Defence Force Academy, Canberra, Special Publication 3.

Armand L. A. 1998. The use of diatom transfer functions in estimating sea-surface temperature and sea-ice in cores from the southeast Indian Ocean. PhD thesis, Australian National University, Canberra (unpubl.).

Baldauf J. G., and Barron J. A. 1991. Diatom biostratigraphy: Kerguelen Plateau and Prydz Bay regions of the Southern Ocean. Proceedings of the Ocean Drilling Program, Scientific Results 119, 547-598.

Bardin V. I., and Belevich A. M. 1985. On the study of early glacial deposits in the Prince Charles Mountains. Antarktika Doklady Komissii 24, 76-81.

Bardin V. I., and Chepaljiga A. L. 1989. Molluscs in early glacial deposits on the shore of Beaver Lake (East Antarctica, Prince Charles Mountains). Antarktika Doklady Komissii 28, 35-38.

Bardin V. I., and Kolosova G. N. 1988. Ancient glacial deposits at Radok Lake. Antarktika Doklady Komissii 27, 66-75.

Barker P. F., Barrett P. J., Cooper A. K., Davey F. J., Domack E. W.,
Escutia C., Kristoffersen Y., and O'Brien P. E. 1998. Ice Sheet history from Antarctic Continental Margin sediments: the ANTOSTRAT approach. Terra Antartica 5, 737-769.

Barrett P. J. 1996. Antarctic paleoenvironments through Cenozoic times: a review. Terra Antartica 3, 103-119.

Barrett P. J., Bleakley N. L., Dickinson W. W., Hannah M. J., and Harper M. A. 1997. Distribution of siliceous microfossils on Mount Feather, Antarctica, and the age of the Sirius Group. In: Ricci C. A. ed. The Antarctic Region; Geological Evolution and Processes; Proceedings of the VII International Symposium on Antarctic Earth Sciences, pp. 763-770. Terra Antartica Publishers, Siena.

Barrett P. J., Fielding C., and Wise S. W. 1998. Initial Report on CRP1, Cape Roberts Project, Antarctica. Terra Antartica 5, 187.

Barron J. A., and Larsen B., and Shipboard Scientific Party. 1991. ODP leg 188. Proceedings of the Ocean Drilling Program Initial Reports 119. Ocean Drilling Program, Texas A\&M University, College Station.

Basile I., Grousset F. E., Revel M., Petit J. R., Biscaye P. E., and Barkov N. I. 1997. Patagonian origin of glacial dust deposited in East Antarctica (Vostok and Dome C) during glacial stages 2, 4 and 6. Earth and Planetary Science Letters 146, 573-589.

Beaver J. 1981. Apparent Ecological Characteristics of Some Common Freshwater Diatoms. Ontario Ministry of the Environment, Ottawa.

Berggren W. A., Kent D. V., Flynn J. J., and Van Couvering J. A. 1985. Cenozoic geochronology. Geological Society of America Bulletin 96, 1407-1418.

Berggren W. A., Kent D. V., Swisher C. C., and Aubry M. P. 1995. A revised Cenozoic geochronology and chronostratigraphy. In: Berggren W. A., Kent D. V., Aubry M. P., and Hardenbol J. A. eds. Geochronology, Time Scales and Global Stratigraphic Correlation, pp. 129-212. Society for Sedimentary Geology Special Publication 54.

Bohaty S. M., Scherer R. P., and Harwood D. M. 1998. Quaternary diatom biostratigraphy and palaeoenvironments of the CRP-1 drillcore, Ross Sea, Antarctica. Terra Antartica 5, 431-453.

Bohaty S. M., Wise S. W. Jr, Duncan R. A., Moore C. L., and Wallace P. J. 2003. Neogene diatom biostratigraphy, tephra stratigraphy, and chronology of ODP Hole 1138A, Kerguelen Plateau. Proceedings of the Ocean Drilling Program, Scientific Results 183; http:// www-Odp.Tamu.Edu/Publications/183 SR/016/016.htm

Bradbury J., and Krebs W. N. 1995. Fossil continental diatoms: paleolimnology, evolution and biochronology. In: Babcock L. E., and Ausich W. I., eds. Siliceous Microfossils (Short Courses in Paleontology) 8, pp. 119-138. Paleontological Society, Knoxville.

Burckle L. H., Gayley R. I., Ram M., and Petit J-R. 1988. Biogenic particles in Antarctic ice cores and the source of Antarctic dust. Antarctic Journal of the United States 23, 71-72.

Burckle L. H., Kellogg D. E., Kellogg T. B., and Fastook J. L. 1997. A mechanism for emplacement and concentration of diatoms in glacigenic deposits. Boreas 26, 55-60.

Cande S. C., and Kent D. V. 1995. Revised calibration of the geomagnetic polarity time scale for the Late Cretaceous and Cenozoic. Journal of Geophysical Research 100, 6093-6095.

Censarek B., and Gersonde R. 2002. Miocene diatom biostratigraphy at ODP Sites 689, 690, 1088, 1092 (Atlantic sector of the Southern Ocean). Marine Micropaleontology 45, 225-268.

Chalmers M. O., Harper M. A., and Marshall W. A. 1996. An Illustrated Catalogue of Airborne Microbiota from the Maritime Antarctic. British Antarctic Survey, Cambridge.

Ciesielski P. F. 1983. The Neogene and Quaternary diatom biostratigraphy of subantarctic sediments, Deep Sea Drilling Project Leg 71. Initial Reports of the Deep Sea Drilling Project 90, 635-665. 
Cramer J. 1979. The algae of Southern Victoria Land, Antarctica. Bibliotheca Phycologica 46, 1-163.

Delmas R. J., and Petit J. R. 1994. Present Antarctic aerosol composition: A memory of ice age atmospheric dust? Geophysical Research Letters 21, 879-882.

Dowdeswell J. A., Whittington R. J., and Marienfeld P. 1994. The origin of massive diamicton facies by iceberg rafting and scouring, Scoresby Sund, East Greenland. Sedimentology 41, 21-35.

Dunbar R. B., Leventer A. R., and Stockton W. L. 1989. Biogenic sedimentation in McMurdo Sound, Antarctica. Marine Geology 85, 155-179.

Fenner J. M. 1991. Late Pliocene-Quaternary quantitative diatom stratigraphy in the Atlantic sector of the Southern Ocean. Proceedings of the Ocean Drilling Program Scientific Results 114, 97-121.

Fielding C. R., and Webb J. A. 1995. Sedimentology of the Permian Radok Conglomerate in the Beaver Lake area of MacRobertson Land, East Antarctica. Geological Magazine 132, 51-63.

Gaudichet A., De Angelis M., Lefevre R., Petit J. R., Korotkevitch Y. S., and Petrov V. N. 1988. Mineralogy of insoluble particles in the Vostok Antarctic ice core over the last climatic cycle (150 kyr). Geophysical Research Letters 15, 1471-1474.

Gaudichet A., Petit J. R., Lefevre R., and Lorius C. 1986. An investigation by analytical transmission electron microscopy of individual insoluble microparticles from Antarctic (Dome C) ice core samples. Tellus 38B, 250-261.

Gersonde R., and Burckle L. H. 1990. Neogene diatom biostratigraphy of ODP Leg 113, Weddell Sea (Antarctic Ocean). Proceedings of the Ocean Drilling Program Scientific Results 113, 761-789.

Gersonde R., Kyte F. T., Bleil U., Diekmann B., Flores J. A., Gohl K., Grahl G., Hagen R., Kuhn G., Sierro F. J., Völker D., Abelmann A., and Bostwick J. A. 1997. Geological record and reconstruction of the late Pliocene impact of the Eltanin asteroid in the Southern Ocean. Nature 27, 357-363.

Gombos A. M., and Ciesielski P. F. 1983. Late Eocene to early Miocene diatoms from the southwestern Atlantic. Initial Reports of Deep Sea Drilling Project 90, 583-634.

Grousset F. E., Biscaye P. E., Revel M., Petit J.-R., Pye K., Joussaume S., and Jouzel J. 1992. Antarctic (Dome C) ice-core dust at 18 k.y. B.P. Isotopic constraints on origins. Earth and Planetary Science Letters 111, 175-182.

Hallegraeff G. M. 1986. Taxonomy and morphology of the marine plankton diatoms Thalassionema and Thalassiothrix. Diatom Research 1, 57-80.

Hambrey M. J., and Dowdeswell J. A. 1994. Flow regime of the Lambert Glacier-Amery Ice Shelf System, Antarctica: structural evidence from satellite imagery. Annals of Glaciology 20, 401-406.

Hambrey M. J., and McKelvey B. C. 2000a. Neogene fjordal sedimentation on the western margin of the Lambert Graben, East Antarctica. Sedimentology 47, 577-607.

Hambrey M. J., and McKelvey B. C. 2000b. Major Neogene fluctuations of the east Antarctic ice sheet: stratigraphic evidence from the Lambert Glacier Region. Geology 28, 887-890.

Harper M. A., and Harwood D. M. 1992. Ancient fresh water diatoms in Antarctica. New Zealand Limnological Society Newsletter 18,26 .

Harris P. T., O’Brien P. E., Quilty P. G., Taylor F., Domack E., De Santis L., and Raker B. 1997. Post Cruise Report, Antarctic CRC Marine Geoscience: Vincennes Bay, Prydz Bay and Mac. Robertson Shelf: AGSO Cruise 186, ANARE Voyage 5, 1996/97 (BRAD). Australian Geological Survey Organisation Record 1997/51.

Harwood D. M. 1986a. Diatom biostratigraphy and paleoecology with a Cenozoic history of Antarctic ice sheets. PhD thesis, Ohio
State University, Columbus (unpubl.).

Harwood D. M. 1986b. Diatoms. In: Barrett P. J. ed. Antarctic Cenozoic History from the MSSTS-1 Drillhole, Mcmurdo Sound, pp. 69109. New Zealand Department of Scientific and Industrial Research Bulletin 237.

Harwood D. M., and Bohaty S. M. 2001. Early Oligocene siliceous microfossil biostratigraphy of Cape Roberts Project CRP-3, Victoria land Basin, Antarctica. Terra Antartica 8, 315-338.

Harwood D. M., and Maruyama T. 1992. Middle Eocene to Pleistocene diatom biostratigraphy of Southern Ocean Sediments from the Kerguelen Plateau. Proceedings of the Ocean Drilling Program, Scientific Results 120, 683-733.

Harwood D. M., McMinn A., and Quilty P. G. 2000. Diatom biostratigraphy and age of the Pliocene Sørsdal Formation, Vestfold Hills, East Antarctica. Antarctic Science 12, 443-446.

Harwood D. M., and Rose S. A. 1998. Report on diatom analysis of Mount Feather COMRAC Cores. In: Wilson G., and Barron J. eds. Mount Feather Sirius Group Core Workshop and Collaborative Sample Analysis, pp. 79-89. Byrd Polar Research Center, Ohio State University, Report 14.

Harwood D. M., Scherer R. P., and Webb P. N. 1989. Multiple Miocene marine productivity events in West Antarctica as recorded in Upper Miocene sediments beneath the Ross Ice Shelf (Site J-9). Marine Micropaleontology 15, 91-115.

Harwood D. M., and Webb P. N. 1998. Glacial transport of diatoms in the Antarctic Sirius Group: Pliocene refrigerator. GSA Today 8, 1-8.

Kellogg D. E., and Kellogg T. B. 1996. Diatoms in South Pole ice: implications for aeolian contamination of Sirius Group deposits. Geology 24, 115-118.

Kellogg T. B., and Kellogg D. E. 2002. Non-marine and littoral diatoms from Antarctic and Subantarctic regions distribution and updated taxonomy. In: Witkowski A. ed. Diatom Monographs, Vol. 1, pp. 1-795. A. R. G. Ganter Verlag K. G., Kommanditgesellschaft, Vaduz.

Krebs K. A. 1997. Mawson Escarpment alpine-type glaciers and the Lambert Glacier Grounding Zone. Proceedings of the Antarctic Cooperative Research Centre Antarctica and Global Change Conference, Abstract 0373. Antarctic CRC, Hobart.

Krebs K. A. 1998. The morphology and dynamics of the Lower Lambert Glacier and Amery Ice Shelf System. MSc thesis, University of Tasmania, Hobart (unpubl.).

Laiba A. A., and Pushina Z. V. 1997. Cenozoic glacial-marine sediments from the Fisher Massif (Prince Charles Mountains). In: Ricci C. A., ed., The Antarctic Region; Geological Evolution and Processes; Proceedings of the VII International Symposium on Antarctic Earth Sciences, pp. 977-984. Terra Antartica Publishers, Siena.

McKelvey B., Hambrey M., Harwood D., Mabin M., Webb P., and Whitehead J. 2001. The Pagodroma Group: the Neogene Record in the Northern Prince Charles Mountains of a dynamic Lambert Glacier and East Antarctic Ice Sheet. Antarctic Science 13, 455-468.

McKelvey B. C., and Stephenson N. C. N. 1990. A geological reconnaissance of the Radok Lake area, Amery Oasis, Prince Charles Mountains. Antarctic Science 2, 53-66.

Mcloughlin S., and Drinnan A. N. 1997. Revised stratigraphy of the Permian Bainmedart Coal Measures, northern Prince Charles Mountains, East Antarctica. Geological Magazine 134, 335-353.

Mikhalsky E. V., Sheraton J. W., Laiba A. A., Tingey R. J., Thost D. E., Kamenev E. N., and Fedorov L. V. 2001. Geology of the Prince Charles Mountains, Antarctica. AGSO - Geoscience Australia Bulletin 247.

Miller M., and Mabin M. C. G. 1998. Antarctic Neogene landscapes: in the refrigerator or in the deep-freeze? GSA Today 8, 1-8. 
Mortlock R. A., and Froelich P. N. 1989. A simple method for the rapid determination of biogenic opal in pelagic marine sediments. Deep-Sea Research 36, 1415-1426.

Naish T. R., Woolfe K. J., Barrett P. J., Wilson G. S., Atkins C., Bohaty S. M., Becker C. J., Claps M., Davey F. J., Dunbar G. B., Dunn A. G., Fielding C. R., Florindo F., Hannah M. J., Harwood D. M., Henerys S. A., Krissek L. A., Lavelle M., Van Der Meer J., Mcintosh W. C., Niessen F., Passchier S., Powell R. D., Roberts A. P., Sagnotti L., Scherer R. P., Strong C. P., Talarico F., Verosub K. L., Villa G., Watkins D. K., Webb P. N., and Wonik T. 2001. Orbital induced oscillations in the East Antarctic ice sheet at the Oligocene/Miocene boundary. Nature 413, 719-723.

O'Brien P. E., and Cooper A. K., and Shipboard Scientific Party. 2001. Proceedings of the Ocean Drilling Program, Initial Reports. 188; http://www-Odp.Tamu.Edu/Publications/188 IR/188ir.htm

Phillips H. A., Allison I., Craven M., Krebs K., and Morgan P. 1996. Ice velocity, mass flux and the grounding line location in the Lambert Glacier-Amery Ice Shelf system, Antarctica. Congress. Proceedings of the 12th Australian Institute of Physics Congress, Paper 0512M. University of Tasmania, Hobart.

Ramsay A. T. S., and Baldauf J. G. 1999. A reassessment of the Southern Ocean Biochronology. Geological Society of London Memoir 18.

Scherer R. P. 1989. Paleoenvironmental studies of the West Antarctic interior: microfossil study of sediment below Upstream B. Antarctic Journal of the United States 22, 35-37.

Scherer R. P. 1991. Quaternary and Tertiary microfossils from beneath Ice Stream B: evidence for a dynamic West Antarctic Ice Sheet history. Palaeogeography, Palaeoclimatology, Palaeoecology 90, 395-412.

Scherer R. P., Aldahan A., Tulaczyk S., Possnert G., Engelhardt H., and Lamb B. 1998. Pleistocene collapse of the West Antarctic Ice Sheet. Science 281, 82-85.

Scherer R. P., Bohaty S. M., and Harwood D. M. 2000. Oligocene and Lower Miocene siliceous microfossil biostratigraphy of Cape Roberts Project Core CRP-2/2A, Victoria Land Basin, Antarctica. Terra Antartica 7, 417-442.

Scherer R., Sjunneskog C., Tulaczyk S., and Aldahan A. 2001. Diatom assemblages and taphonomy in Antarctic diamictons provide constraints for modeling past marine ice sheet configurations and processes. Quaderni di Geofisica 16, 169-170.

Stilwell J. D., Harwood D. M., and Whitehead J. M. 2002. Mid-Tertiary macroinvertebrate-rich clasts from the Battye Glacier Formation, Prince Charles Mountains, East Antarctica. Antarctic Science 14, 69-73.

Stroeven A. P., Prentice M. L., and Kleman J. 1996. On marine microfossil transport and pathways in Antarctica during the late Neo- gene: evidence from the Sirius Group at Mount Fleming. Geology 24, 727-730.

Tingey R. J. (Editor) 1991. Geology of Antarctica. Clarendon Press, Oxford.

Tulaczyk S., Kamb B., Scherer R. P., and Engelhardt H. F. 1998. Sedimentary processes at the base of a West Antarctic ice stream: constraints from textural and compositional properties of subglacial debris. Journal of Sedimentary Research 68, 487-496.

Webb P. N., Harwood D. M., McKelvey B. C., Mercer J. H., and Stott L. D. 1983. Late Neogene and older Cenozoic microfossils in high elevation deposits of the Transantarctic Mountains: evidence for marine sedimentation and ice volume variation on the east Antarctic craton. Antarctic Journal of the United States 18, 96-97.

Whitehead J. M., Harwood D. M., and McMinn A. 2003. Ice-distal late Miocene marine strata from inland Antarctica. Sedimentology 50, 531-552.

Whitehead J. M., and McKelvey B. C. 2001. The stratigraphy of the Pliocene-lower Pleistocene Bardin Bluffs Formation, Amery Oasis, northern Prince Charles Mountains, Antarctica. Antarctic Science 13, 79-86.

Whitehead J. M., Quilty P. G., Harwood D. M., and McMinn A. 2001. Early Pliocene paleoenvironment of the Sørsdal Formation, Vestfold Hills, based on diatom data. Marine Micropaleontology 41, 125-152.

Winter D. M. 1995. Upper Neogene diatom biostratigraphy from coastal drillcores in Southern Victoria Land, Antarctica. MS thesis, University of Nebraska-Lincoln, Lincoln (unpubl.).

Winter D. M., and Harwood D. M. 1997. Integrated diatom biostratigraphy of late Neogene drillholes in Southern Victoria Land and correlation to Southern Ocean records. In: Ricci C. A. ed. The Antarctic Region; Geological Evolution and Processes; Proceedings of the VII International Symposium on Antarctic Earth Sciences, pp. 985-992. Terra Antartica Publishers, Siena.

Winter D., and Iwai M. 2002. Data report: Neogene diatom biostratigraphy, Antarctic Peninsula Pacific margin, ODP Leg 178 rise sites. Proceedings of the Ocean Drilling Program, Scientific Results 178; http://www-Odp.Tamu.Edu/Publications/178 SR/Chap 29/Chap_29.htm

Yanagisawa Y., and Akiba F. 1998. Refined Neogene diatom biostratigraphy for the Northwest Pacific around Japan, with an introduction of code numbers for selected diatom biohorizons. Journal of the Geological Society of Japan 104, 395-414.

Zielinski U., and Gersonde R. 2002. Plio-Pleistocene diatom biostratigraphy from ODP Leg 177, Atlantic sector of the Southern Ocean. Marine Micropaleontology 45, 225-268.

\section{Supplementary Papers (on following pages)}

Appendix 1: Siliceous microfossil taxonomy.

Appendix 2: Size and preservation data from select Pagodroma Groups samples. 
Diatom biostratigraphy of the Cenozoic glaciomarine Pagodroma Group, northern Prince Charles Mountains, East Antarctica*

J. M. WHITEHEAD ${ }^{1,2 \dagger}$, D. M. HARWOOD², B. C. McKELVEY ${ }^{3}$, M. J. HAMBREY $^{4}$ AND A. MCMINN ${ }^{1}$

${ }^{1}$ Institute of Antarctic and Southern Ocean Studies, University of Tasmania, Tas. 7005, Australia.

${ }^{2}$ Department of Geosciences, University of Nebraska-Lincoln, Lincoln, NE, USA.

${ }^{3}$ Division of Earth Sciences, University of New England, Armidale, NSW 2351, Australia.

${ }^{4}$ Centre for Glaciology, Institute of Geography and Earth Sciences, University of Wales, Aberystwyth, Ceredigion SY23 3DB UK.

\section{SUPPLEMENTARY PAPERS}

Australian Journal of Earth Sciences (2004) 51, 521-547

* Appendices 1 and 2 [indicated by an asterisk $\left({ }^{*}\right)$ in the text and listed at the end of the paper] are Supplementary Papers; copies may be obtained from the Geological Society of Australia's website (www.gsa.org.au) or from the National Library of Australia's Pandora archive (http://nla.gov.au/nla.arc25194).

† Corresponding author: jason.whitehead@utas.edu.au

Appendix 1: Siliceous microfossil taxonomy

Appendix 2: Size and preservation data from select Pagodroma Groups samples. 


\section{APPENDIX 1: SILICEOUS MICROFOSSIL TAXONOMY}

Taxa preceded by the symbol * have only been recorded in previous Pagodroma Group studies.

Bacillariophyceae

*Achnanthes brevipes Agardh 1824; Roberts and McMinn 1999, p. 15, pl. 1, figs. 3-4; Harwood et al. 2000, p. 459, figs 9i-j (as Achnanthes charcotii).

Actinocyclus actinochilus (Ehrenberg) Simonsen 1982; Harwood and Maruyama 1992, p. 699, pl. 12, fig. 11; (Figure 6.1).

Actinocyclus ingens Rattray 1890; Harwood and Maruyama 1992, p. 700, pl. 8, fig. 10; pl. 11, fig. 4, 6; pl. 12, fig. 8; (Figure 6.2).

Actinocyclus ingens var. nodus Baldauf in Baldauf and Barron 1980;

Baldauf and Barron 1980, p. 104, pl. 1, fig. 1-9; (Figures 6.3-4).

Actinocyclus aff. ingens Rattray 1891; Schrader 1973, p. 663, pl. 11, fig. 6,7 .

Notes: This diatom is thought to be more common in the Miocene (Schrader 1973).

Actinocyclus karstenii Van Heurck 1909; Harwood and Maruyama 1992, p. 700, pl. 13, fig. 1, 2, 6-6, 10, 11, 13; (Figure 6.6).

Actinocyclus octonarius var. asteriscus Barron 1975; Harwood et al. 2000, p. 459 , pl. 7, fig I.

Actinoptycus senarius Ehrenberg 1838; Gombos 1976, p. 655, pl. 26, fig. 13; (Figure 6.25).

Actinoptycus sp. A; (Figure 6.24).

Actinoptycus spp. Ehrenberg 1843.

Amphora sp. A of Roberts and McMinn 1999, p. 16, pl. 1, figs. 6-7.

Amphora spp. (Ehrenberg) Kützing 1844.

Anaulus sp. Ehrenberg 1844.

Astromphalus spp. Ehrenberg 1844.

Aulacoseira sp.; (Figure 6.18-19). 
Azpeitia tabularis (Grunow) Fryxell and Sims 1986; Akiba 1982, p. 42, pl. 2, fig. 6a, 9.

\section{Caloneis sp. A.}

Chaetoceros lorenzianus Grunow (Dicladia pylea Hanna and Grant); Harwood and Maruyama 1992, pl. 18, fig. 11.

${ }^{*}$ Chaetoceros mitra (Bailey) Cleve.

${ }^{*}$ Chaetoceros subsecundus (Grunow) Hustedt.

Chaetoceros sp. A Harwood and Maruyama 1992, pl.19, figs. 9-10.

Chaetoceros spp. Ehrenberg 1844.

Cocconeis costata Gregory 1855; Roberts and McMinn 1999, p. 19, pl. 2, fig. 5 .

Cocconeis fasciolata (Ehrenberg) Brown 1920; Roberts and McMinn 1999, p. 20 , pl. 2, fig. 4.

Cocconeis pinnata Gregory ex Greville 1859; Roberts and McMinn 1999, p. 20, pl. 2, fig. 6 .

Cocconeis sp. A of Roberts and McMinn 1999, p. 21, pl. 2, figs. 7-10.

Coscinodiscus oculoides Karsten 1905

Coscinodiscus oculus-iridus Ehrenberg 1839; Harwood 1989, p. 78, pl. 1, fig. 1.

Coscinodiscus radiatus Ehrenberg 1939; Harwood 1989, p. 78, pl. 1, fig. 5 Coscinodiscus marginatus Ehrenberg, Hustedt 1930; Barron 1981, p. 118, pl. 1, fig. 2; (Figure 6.8).

Coscinodiscus sp. A; (Figure 6.20).

Coscinodiscus spp. Ehrenberg 1838.

Cyclotella spp. Kützing 1834.

Dactyliosolen antarcticus Castracane 1886.

Denticulopsis delicata Yanagisawa and Akiba 1990, pl 7, figs. 1-4; (Figure 5.8).

Denticulopsis dimorpha (Schrader) Simonsen 1979; Yanagisawa and Akiba 1990, p. 254-255, pl. 4, figs. 42-49; pl. 7, figs. 14-16; (Figures 5.2-3). 
Denticulopsis hustedtii (Simonsen and Kanaya) Simonsen 1979;

Yanagisawa and Akiba 1990, p. 246-248, pl. 3, figs. 15-16; pl. 11, figs. 11-13;

(Figure 5.1).

Denticulopsis ovata (Schrader) Yanagisawa and Akiba 1990; Harwood and Maruyama 1992, p. 702-703, pl. 6, figs. 1-4; pl. 7, figs. 1-4, 6-9, 11-13; pl. 9, figs. 1-4,10-14; pl. 10, fig. 7 (as Denticulopsis meridonalis Maruyama in Harwood and Maruyama 1992); (Figure 5.5).

Denticulopsis praedimorpha Barron ex Akiba 1982; (Figure 5.6); Harwood and Maruyama 1992, p. 703, pl. 6., figs. 8-10, 12, 14-17.

Denticulopsis simonsenii Yanagisawa and Akiba 1990, p. 242-243, pl. 3 , figs. 1-3; pl. 11, figs. 1-5; (Figure 5.7).

Notes: Denticulopsis simonsenii was separated from Denticulopsis vulgaris using the species concepts illustrated and described by Yanagisawa and Akiba (1990). Denticulopsis simonsenii is characterised by more and 2 full striae of aerolae between the psuedosepta, whilst $D$. vulgaris has incomplete rows of areolation on either side (and near) each psuedosepta.

Denticulopsis vulgaris (Okuno) Yanagisawa and Akiba 1990, p. 243-244, pl. 3, figs. 4-8; pl. 11, figs. 6-10.

Notes: See notes under Denticulopsis simonsenii.

Denticulopsis spp. (Simonsen) Akiba and Yanagisawa 1990.

Diploneis sp. A.

Diploneis frickei (Van Heurck) Heiden and Kolbe 1958; Harwood et al. 2000, p. 459 , pl. 9 , fig m.

*Diploneis oculata (Brébisson) Cleve 1894-1895.

Diploneis subovalis Cleve 1894; Harwood et al. 2000, p. 459, pl. 9, fig. I. *Diploneis smithii (Brébisson) Cleve 1894-1895.

Diploneis bomboides (Schmidt) Cleve 1894-1895; Roberts and McMinn 1999, p. 21, pl. 3, fig 1, (as Diploneis splendidus (Gregory) Cleve 18941895).

Eucampia antarctica (Castracane) Mangin 1914; (Figure 6.7).

${ }^{\star}$ Eunotia sp. Ehrenberg 1837.

Fragilaria spp. Lyngbye 1819. 
Fragilariopsis curta (Van Heurck) Hasle 1958; Harwood and Maruyama 1992, p. 704, pl. 17, figs. 1-4 (as Nitzschia curta (Van Heurck) Hasle 1972).

Fragilariopsis interfrigidaria (McCollum) Gersonde and Bárcena 1998;

Baldauf and Barron 1991, p. 589, pl. 7, fig. 12 (as Nitzschia interfrigidaria McCollum 1975); (Figure 5.9).

Fragilariopsis kerguelensis (O'Meara) Hasle 1952; Medlin and Priddle 1990, p. 181, pl. 24.2, fig. 11-18; p. 187, pl. 24.3, fig. 9 (as Nitzschia kerguelensis (O’Meara) Hasle 1972); (Figure 5.11; Figure 6.15).

Fragilariopsis praeinterfrigidaria (McCollum) Gersonde and Bárcena 1998; Barron 1985, p. 805, fig. 14, fig. 5,6 (as Nitzschia praeinterfrigidaria McCollum 1975); (Figure 5.9).

*Fragilariopsis sublinearis (Van Heurck) Heiden 1928.

Fragilariopsis spp. Hustedt 1913.

Gomphonema angustatum var. A; (Figure 5.14).

Synonym: Navicula sp. in Winter 1995, pl. 7, figs. 2-4.

Gomphonema angustatum var. B; (Figure 5.15).

Grammatophora spp. Ehrenberg 1840.

Hyalodiscus valens Schmidt 1888; Harwood et al. 2000, p. 459, pl. 8, fig. h. *Hyalodiscus obsoletus Sheshukova.

Hyalodiscus zonulatus Peragallo 1921; Harwood et al. 2000, p. 459, pl. 8, fig. I.

*Hyalodiscus sp. Ehrenberg 1845.

Isthmia sp. Agardh 1832.

Melosira sp. Agardh 1824.

Notes: possibly recorded as ${ }^{*}$ Podosira sp. by Bardin and Belevich (1985).

*Navicula cryophila Manguin 1957.

Navicula directa (Smith) Ralfs in Pritchard 1861; Roberts and McMinn 1999, p. 31, pl. 4, fig. 13.

*Navicula glaciei Van Heurck 1909.

Synonym: *Navicula gelida var. parvula Heiden and Kolbe 1928. 
Notes: synonym recorded as a separate species in Bardin and Belevich (1985) with an abundance of 1-5 specimens per field-of-view. The N. glaciei abundance has been altered to 1-10 to accommodate this.

*Navicula retusa Donkin.

Synonym: Navicula cancellata var. retusa (Brébisson) Cleve.

*Navicula spp. Bory 1822.

*Nitzschia acicularis (Kützing) Smith 1853.

*Nitzschia cholonii Van Heurck?

*Nitzschia delicatissima Cleve.

*Nitzschia frustulum (Kützing) Grunow.

*Nitzschia scabra Cleve.

*Nitzschia sp. A as in Harwood 1989.

Nitzschia reinholdii Kanaya ex Barron and Bauldauf 1986; Gersonde and

Burckle 1990, p. 782 pl. 2, fig. 1; (Figure 5.10).

Nitzschia sp. Hassall 1845.

*Odontella weissflogii (Janisch) Grunow.

Paralia sulcata (Ehrenberg) Cleve 1873; Harwood and Maruyama 1992, p.

704, pl. 1, fig. 9

*Paralia sp. Heiberg 1863.

Pinnularia quadratea (Schmidt) Cleve 1895; Harwood et al. 2000, p. 460, pl. 10, fig, $\mathrm{f}$.

\section{Pinnularia sp. A.}

*Porosira antarctica Kozlova 1962.

Notes: possible synonym to Porosira glacialis (Grunow) Jørgensen; however, Porosira antarctica is considered to be smaller and usually weakly silicified (Priddle and Fryxell 1985).

*Porosira pseudodenticulata (Hustedt) Jousé 1962.

Proboscia barboi (Brün) Jordan and Priddle 1991; Harwood and Maruyama, 1992, p. 706, pl. 11, fig. 13.

Pyxilla sp. (Pyxilla reticulata Grove and Sturt 1887); (Figure 5.3).

Synonym: Fenner 1985 notes that $P$. prolongata Brun and $P$. johnsoniana Forti 1909 are probably synonyms of $P$. reticulata. 
Notes: The Pyxilla sp. fragments appear to be $P$. reticulata and can be differentiated from other Pyxilla spp. (or Pyrgupyxis spp.). Pyxilla reticulata has finer areolae 3-6 in $10 \mu \mathrm{m}$ (Fenner 1985), whilst $P$. oligocenica Jousé has coarser areolae: 2-3 in $10 \mu \mathrm{m}$ (Fenner 1985) and $P$. gracilis Tempère et Forti has coarser areolae, 2-3 in $10 \mu \mathrm{m}$ (Fenner 1985), and a smoother valve surface (Gombos 1987, p. 798, pl. 1, fig. 5; Fourtanier 1991, p. 186, pl. 4, fig. 10). Pyrgupyxis. eocena Hendy has a distinctly different shape to $P$. reticulata (Gombos 1976, p. 647, pl. 22, figs 6-7; Harwood and Bohaty 2001, p. 330, pl. 4, fig. 5. Pyxilla reticulata is the only species within the Pyxilla genus identified from marine strata in Prydz Bay (Barron and Mahood 1993).

Rhabdonema japonica group Tempère and Brün in Brün and Tempère 1889; Harwood et al. 2000, p. 460, pl. 9, fig. K; (Figure 5.16).

${ }^{\star}$ Rhabdonema sp. cf. R. elegans Tempère and Brün.

Rhabdonema spp. Kützing 1844

Rhaphoneis spp. Ehrenberg 1845; (e.g. Figure 5.19).

Rhizosolenia hebetata group Bailey 1856; (Figure 6.17); Koizumi, 1973, p. 833, pl. 5, fig. 35 .

Notes: This group consists of Rhizosolenia spp. lacking otaria.

Rhizosolenia styliformis group Brightwell 1858; Harwood and Maruyama 1992, p. 705, pl. 18, fig. 20.

Notes: This group consists of Rhizosolenia spp. with otaria.

Rouxia heteropolara Gombos 1974; (Figure 5.18); Gersonde and Burckle 1990, p. 782, pl. 5, fig. 2.

Rouxia isopolica Schrader 1976, p. 635-636, pl. 5, figs. 9, 14-15, 20. (Figure 5.17).

Rouxia naviculoides Schrader 1973, p. 710, pl. 5, figs. 27-32.

Rouxia spp. Brün and Heribaud 1893

Stellarima microtrias (Ehrenberg) Hasle and Sims 1986; (Figure 6.9); Harwood 1989, p. 80 pl. 1, fig. 4.

Stellarima stellaris (Roper) Hasle and Sims 1986; (Figure 6.9); Harwood 1989, p. 80, pl. 1, fig. 3.

*Stellarima spp. Hasle and Sims 1986. 
Stephanodiscus spp. Ehrenberg 1845; (Figure 6.18).

Stephanopyxis grunowii Grove and Sturt 1888; Harwood, 1989, p. 81, pl. 2, fig. 1, 2-4.

Stephanopyxis splendidus (Greville) Harwood 1989; (Figure 6.10);

Harwood 1989, p. 81, pl. 2, fig. 1, 2-4.

Stephanopyxis turris (Greville and Arnott) Ralfs, in Pritchard 1861; (Figure 6.11); Harwood, 1989, p. 81, pl. 2, fig. 21-23.

Stictodiscus hardmanius Greville 1865; Harwood 1989, p. 81, pl. 1, fig. 6. Syndera sp. Ehrenberg 1832.

Synedropsis spp. Hasle et al.

Tabellaria fenestrata (Lyngb.) Kützing 1844; Contant and Duthie 1978, p 43 pl. XV figs. 4-7.

Tabellaria sp. Ehrenberg ex Kützing 1844.

Thalassionema nitzschioides group Grunow, in Van Heurck; (Figure 5.12). Thalassionema nitzschioides var. parva Heiden 1928; Koizumi and Tanimura 1982, p. 300, pl. 6, fig. 11.

*Thalassiosira antarctica Comber 1896.

Thalassiosira gracilis var. gracilis (Karsten) Hustedt 1958; (Figure 6.23); Johansen and Fryxell 1985, p. 168-170, fig 58, 59.

Thalassiosira insigna (Jousé) Harwood and Maruyama 1991; Harwood and Maruyama 1992, p. 707, pl. 14, fig. 3-5.

Thalassiosira inura Gersonde 1991; Harwood and Maruyama 1992, p. 707, pl. 5, fig. 14; pl. 14, fig. 12-14, 16.

Thalassiosira nativa Sheshukova-Poretzkaya 1959; Baldauf and Barron 1991, p. 597, pl. 6, fig 5.

Notes: appears like $T$. inura, but differentiated in that the central haline area is smaller in $T$. nativa.

Thalassiosira oliverana (O'Meara) Makarova and Nikolaev 1983; (Figure 6.12); Abbott 1974, p. 319, pl. 2, fig. D-F.

*Thalassiosira oestrupii (Ostenfeld) Hasle 1972.

Thalassiosira spp. Cleve 1873.

Thalassiothrix sp. Cleve and Grunow 1880. 
Trachyneis aspera (Ehrenberg) Cleve 1984; Roberts and McMinn 1999, p. 44, pl. 7, fig. 9.

*Triceratium oamaruense var. sparsipunctatum Grove.

Trinacria excavata Heiberg 1863; (Figure 6.22); Harwood 1989, p. 82, pl. 3, fig. 1.

${ }^{\star T}$ Trigonium arcticum (Brightwell) Cleve 1868.

Gen. et sp. B of Harwood 1986a.

Dinophyceae

Actiniscus pentasteris (Ehrenberg) Ehrenberg 1854.

Dictyochophyceae

Distephanus spp. Stoehr 1880; (Figure 6.16).

Ebriophyceae

Pseudammodochium lingii Bohaty et Harwood 2000; (Figure 6.21). 
Appendix 2. Size and preservation data from select Pagodroma Groups samples.

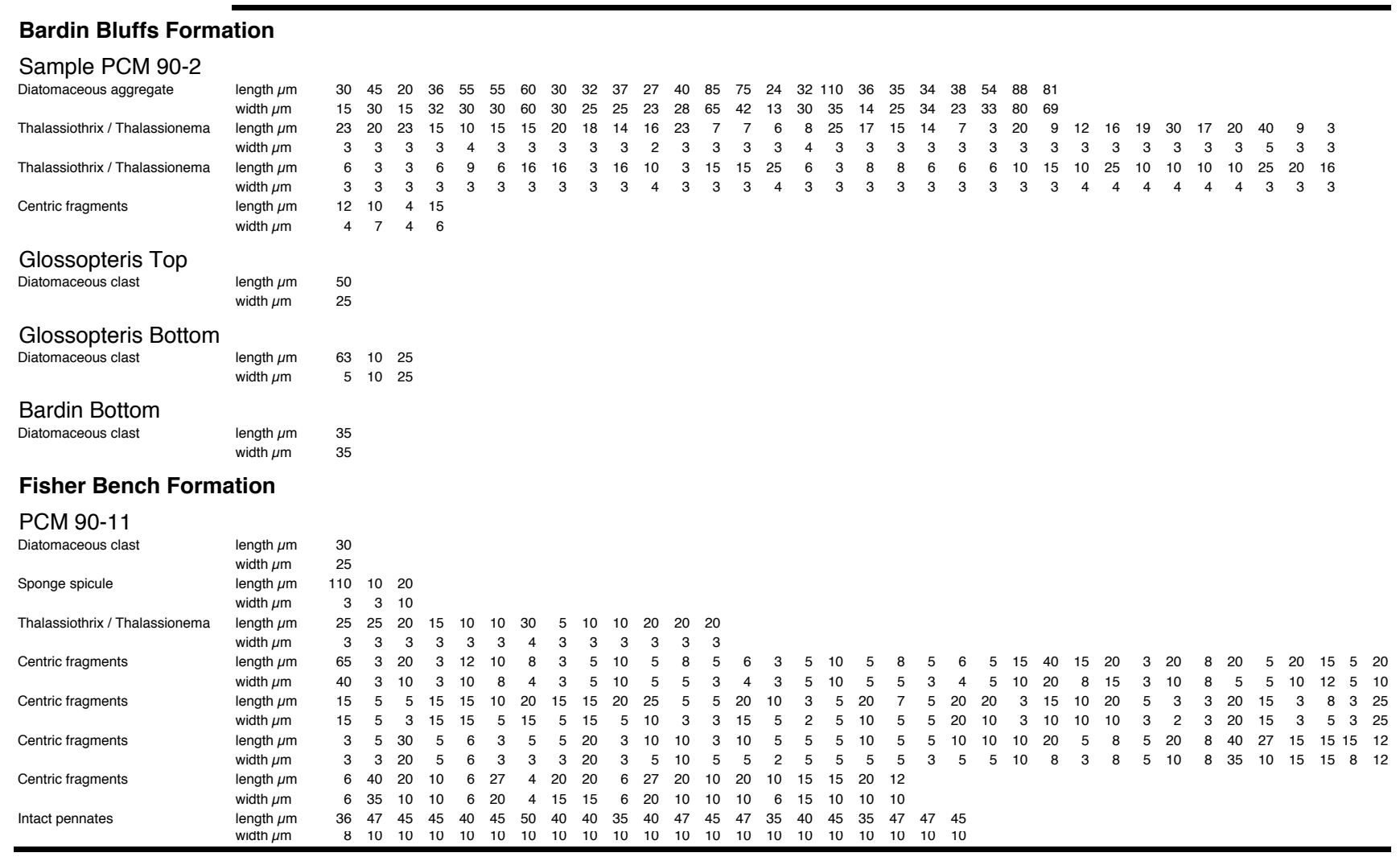

